\title{
CELLS AND THE REFLECTION REPRESENTATION OF WEYL GROUPS AND HECKE ALGEBRAS
}

\author{
J. MATTHEW DOUGLASS
}

\begin{abstract}
Let $\mathscr{H}$ be the generic algebra of the finite crystallographic Coxeter group $W$, defined over the ring $\mathbb{Q}\left[u^{1 / 2}, u^{-1 / 2}\right]$. First, the two-sided cell corresponding to the reflection representation of $\mathscr{H}$ is shown to consist of the nonidentity elements of $W$ having a unique reduced expression. Next, the matrix entries of this representation are computed in terms of certain KazhdanLusztig polynomials. Finally, the Kazhdan-Lusztig polynomials just mentioned are described in case $W$ is of type $\mathrm{A}_{l-1}$ or $\mathrm{B}_{l}$.
\end{abstract}

\section{INTRODUCTION}

Let $(W, S)$ be a finite crystallographic Coxeter system and let $\mathscr{H}$ be the Hecke algebra of $(W, S)$, defined over the ring $A=\mathbb{Q}\left[u^{1 / 2}, u^{-1 / 2}\right]$. Then $\mathscr{H}$ is free as an $A$-module with "standard" basis, $\left\{T_{x} \mid x \in W\right\}$, and if we denote the length function on $W$ by $\ell(\cdot)$, the multiplication in $\mathscr{H}$ is determined by the formulas

$$
T_{s} T_{x}= \begin{cases}T_{s x} & \text { if } \ell(s x)>\ell(x), \\ (u-1) T_{x}+u T_{s x} & \text { if } \ell(s x)<\ell(x)\end{cases}
$$

$(s \in S, x \in W)$. In this paper we will address the following problems concerning the reflection representation of $\mathscr{H}$ :

Describe the left, right, and two-sided cells of $W$ having the property that the corresponding ideals in $\mathbb{Q}\left(u^{1 / 2}\right) \otimes_{A} \mathscr{H}$ contain the reflection representation.

Describe explicitly the matrix entries of the reflection representation.

In order to state our results, we need some more notation. Let $\leq_{L}$ and $\leq_{L R}$ be the preorders defined by Kazhdan and Lusztig in [6] and let $\sim_{L}$ and $\sim_{L R}$ be the corresponding equivalence relations. The $\sim_{L}$ equivalence classes are called left cells and the $\sim_{L R}$ equivalence classes are called two-sided cells.

Let $K=\mathbb{Q}\left(u^{1 / 2}\right)$ be the quotient field of $A$. Following Lusztig [8], we obtain decompositions of $K \otimes_{A} \mathscr{H}$ corresponding to the various partitions of $W$ into

Received by the editors March 16, 1989.

1980 Mathematics Subject Classification (1985 Revision). Primary 20G05. 
cells: Let $\left\{C_{x} \mid x \in W\right\}$ be the basis of $\mathscr{H}$ described in [6, Theorem 1.1]. For $x, y, z \in W$, define elements $h_{x, y, z}$ in $A$ by

$$
C_{x} C_{y}=\sum_{z \in W} h_{x, y, z} C_{z}
$$

It follows from [6, Theorem 1.1] that $h_{x, y, z}$ is symmetric in $u^{1 / 2}$ and $u^{-1 / 2}$.

Let $\mathscr{M}$ be a free $A$-module with basis $\left\{c_{x} \mid x \in W\right\}$. Then as in [8], the formulas

$$
C_{x} c_{y}=\sum_{z \sim L R y} h_{x, y, z} c_{z} \text { and } c_{x} C_{y}=\sum_{z \sim L R} h_{x, y, z} c_{z}
$$

define a two-sided action of $\mathscr{H}$ on $\mathscr{M}$ so that $K \otimes_{A} \mathscr{H} \cong K \otimes_{A} \mathscr{M}$ as twosided $\mathscr{H}$-modules. For a subset, $Y$, of $W$ define $\mathscr{M}_{Y}$ to be the $A$-submodule of $\mathscr{M}$ with basis $\left\{c_{y} \mid y \in Y\right\}$. From (1.5) we have $\mathscr{M} \cong \bigoplus \mathscr{M}_{X}$ (sum over all two-sided cells, $X$ ) as two-sided $\mathscr{H}$-modules. It follows from [8, Property (A)] that for a two-sided cell, $X$, we have

$$
K \otimes_{A} \mathscr{M}_{X} \cong \bigoplus\left(K \otimes_{A} \mathscr{M}_{\Gamma}\right)
$$

as left $\mathscr{H}$-modules, where the sum is over all left cells, $\Gamma$, with $\Gamma \subseteq X$.

Curtis, Iwahori, and Kilmoyer [3] construct an irreducible representation of $\mathscr{H}$, called the reflection representation. Their construction may be summarized as follows: Let $\Delta$ be a root system associated to $W$, and for $s \in S$ let $\alpha_{s}$ be the corresponding simple root. Let $\widetilde{E}$ be a free $A$-module with basis $\left\{d_{t} \mid t \in S\right\}$. Define an action of $T_{s}$, for $s \in S$, on $\widetilde{E}$ by the formulas

$$
T_{s} d_{t}= \begin{cases}-d_{s} & \text { if } s=t, \\ u d_{t}-u^{1 / 2}\left\langle\alpha_{t}, \alpha_{s}\right\rangle d_{s} & \text { if } s \neq t,\end{cases}
$$

where $\left\langle\alpha_{\hat{t}}, \alpha_{\hat{s}}\right\rangle$ is the Cartan integer $2\left(\left(\alpha_{t}, \alpha_{s}\right) /\left(\alpha_{s}, \alpha_{s}\right)\right)$. It is shown in [3] that (1.6) defines an irreducible representation of $\mathscr{H}$.

Since $K \otimes_{A} \mathscr{H} \cong K \otimes_{A} \mathscr{M}$ as two-sided $K \otimes_{A} \mathscr{H}$-modules and $\mathscr{M} \cong \bigoplus \mathscr{M}_{X}$ (sum over all two-sided cells, $X$ ) as two-sided $\mathscr{H}$-modules, it follows that for each two-sided cell, $X$, of $W, K \otimes_{A} \mathscr{M}_{X}$ is isomorphic to a two-sided ideal of $K \otimes_{A} \mathscr{H}$. Thus there is a unique two-sided cell, $X$, with the property that $K \otimes_{A} \widetilde{E}$ is a constituent of $K \otimes_{A} \mathscr{M}_{X}$. In $\S 2$ we will show that with $\widetilde{E}$ as above, $X$ is characterized by the following equivalent properties (see [2.5]):

(1) $a(X)=1$, where $a(\cdot)$ is the function on $W$, constant on two-sided cells, defined by Lusztig in [11] (see $\S 2$ ),

(2) $X=\bigcup_{s \in S} \Gamma_{s}$, where for $s \in S, \Gamma_{s}$ is the left cell containing $s$,

(3) $X$ is precisely the set of nonidentity elements of $W$ having a unique reduced expression.

It follows from the formulas for the generic degree of the reflection representation given in [3] that the reflection representation is special, as defined by Lusztig [10, 4.1.4]. Hence, by the results in [10, Chapter 5], we see that the 
reflection representation appears with multiplicity one in each $\mathscr{M}_{\Gamma_{s}}$. In order to give a complete answer to (1.2), it remains to give reduced expressions for the elements in $X$. This is done in $\S 4$.

Define the "matrix entries," $\pi(x, s, t)$, for $x \in W$ and $s, t \in S$, of the reflection representation by the formula

$$
T_{x} d_{s}=\sum_{t \in S} \pi(x, s, t) d_{t} .
$$

Our answer to (1.3) consists of two parts. In $\S 2$ we give a formula for the $\pi(x, s, t)$ 's in terms of certain Kazhdan-Lusztig polynomials (see [2.8]). In $\S 5$ and $\S 6$ we compute these Kazhdan-Lusztig polynomials when $W$ is of type $\mathrm{A}_{l-1}$ or $\mathrm{B}_{l}$, and hence we obtain "explicit" formulas for the $\pi(x, s, t)$ 's in these cases. The methods we use should easily extend to give explicit formulas for the $\pi(x, s, t)$ 's in the other cases as well. Some of the Kazhdan-Lusztig polynomials given in $\S 6$ have been previously computed by Boe [2], using different methods.

When $W$ is of classical type, the general form of the matrix entries has been obtained by Tiwari [14], but no method is given to compute them.

Recall that $\Delta$ is a root system for $W$. One of the important properties of root systems is that if $w \in W$, and $\alpha \in \Delta$, then when $w(\alpha)$ is expressed in terms of a base, the coefficients are either all nonnegative or all nonpositive integers. By combining our formula $(2.8)$ for the $\pi(x, s, t)$ 's with a positivity result for certain differences of Kazhdan-Lusztig polynomials, we obtain a proof of the following generalization of this property:

For $x \in W$ and $s \in S$, the coefficients of the matrix entries $\pi(x, s, t)$, $t \in S$, are all of the same sign as $\ell(x s)-\ell(x)$.

The details of the proof are given in [5]. This result was conjectured by Lusztig, and proved, using a case-by-case analysis, when $W$ is of classical type, by Tiwari [14]. The proof in [5] is valid for all types of Weyl groups and does not rely on a case-by-case analysis.

This paper is organized as follows: In $\S 2$ we characterize the two-sided cell, $X$, corresponding to the reflection representation, and prove (2.8). The results concerning Weyl groups of type $\mathrm{A}_{l-1}$ and $\mathrm{B}$, which we will need later are collected in $\S 3$. In $\S 4$, reduced expressions for all the elements of $X$ are described and the $n_{w}$ of (2.8) are computed. In $\S 5$, the Kazhdan-Lusztig polynomials of (2.8) are computed when $W$ is of type $\mathrm{A}_{l-1}$, and the results of the preceding sections are applied to give simple expressions for the $\pi(x, s, t)$ 's in this case (see [5.4]). We also derive a formula, due to Tiwari [14], for the diagonal entries $\pi(x, s, s)$ (see [5.8]). Finally, in $\S 6$ we compute the Kazhdan-Lusztig polynomials of (2.8) when $W$ is of type $\mathrm{B}_{l}$, and we calculate the matrix entries, $\pi(x, s, t)$, in this case.

For $s$ in $S$ let $W_{(s)}$ denote the (maximal) parabolic subgroup of $W$ generated by $S-\{s\}$. It is a perhaps surprising consequence of the calculations in 
$\S 5$ and $\S 6$ that for $x$ in $W$ and $s, t$ in $S$, the matrix entry, $\pi(x, s, t)$, depends on the structure of the Bruhat poset of $\left(W_{(t)}, W_{(s)}\right)$-double cosets.

\section{Cells and the Reflection Representation}

In this section we characterize the cells corresponding to the reflection representation of $\mathscr{H}$. We also show that the matrix coefficients of this representation can be computed in terms of Kazhdan-Lusztig polynomials (this is the content of [2.8]).

We will continue the notation of $\S 1$. In addition, $\leq$ will be the Bruhat order; $\leq_{R}$ will be the preorder on $W$ defined by

$$
x \leq_{R} y \quad \text { if and only if } \quad x^{-1} \leq_{L} y^{-1},
$$

for $x, y \in W$; and $w_{0}$ will denote the element in $W$ with maximal length. Given $x \in W$, we will write $u_{x}$ for $u^{\ell(x)}$. We also define $\mathscr{L}(x)=\{s \in S \mid$ $s x<x\}$ and $\mathscr{R}(x)=\mathscr{L}\left(x^{-1}\right)$.

We will assume that the reader is familiar with Kazhdan-Lusztig polynomials. Let $y, w \in W$. Define $\mu(y, w)$ as follows: If $y<w$, then $\mu(y, w)$ is the coefficient of $u^{(\ell(w)-\ell(y)-1) / 2}$ in $P_{y, w}$. If $w<y$, then $\mu(y, w)=\mu(w, y)$ (which is well-defined by the above). Otherwise, define $\mu(y, w)=0$. As in [10, 5.1.12], for $s \in S$ and $w \in W$, we have

$$
C_{s} C_{x}= \begin{cases}-\left(u^{1 / 2}+u^{-1 / 2}\right) C_{x} & \text { if } s \in \mathscr{L}(x), \\ C_{s x}+\sum_{\substack{y<x \\ s y<y}} \mu(y, x) C_{y} & \text { if } s \notin \mathscr{L}(x),\end{cases}
$$

and a similar formula for $C_{w} C_{s}$.

In [9], Lusztig defines a correspondence between irreducible $\mathbb{Q} W$-modules and two-sided cells. In general, given an irreducible $\mathbb{Q} W$-module, $E$, there is a unique two-sided cell, $X$, so that $E$ is a constituent of $\mathbb{Q} \otimes_{A} \mathscr{M}_{X}$ (here we consider $\mathbb{Q}$ as an $A$-module via the specialization $\left.u^{1 / 2} \mapsto 1\right)$.

In order to determine the cells corresponding to the reflection representation of $W$, we will need the function $a: W \rightarrow \mathbb{N}$ defined in [11]. Specifically, for $z \in W$, define $a(z)=\max \left\{\operatorname{deg}_{1 / 2} h_{x, y, z} \mid x, y \in W\right\}$, where $\operatorname{deg}_{1 / 2}$ means degree in $u^{1 / 2}$ and $h_{x, y, z}$ is as in (1.4). Since $h_{x, y, z}$ is symmetric in $u^{1 / 2}$ and $u^{-1 / 2}$, it follows that $a(z) \in \mathbb{N}$. Define $\delta(z)$ to be the degree, in $u$, of $P_{1, z}$ and let

$$
\mathscr{D}=\{z \in W \mid a(z)=\ell(z)-2 \delta(z)\} .
$$

Elements of $\mathscr{D}$ are called Duflo involutions. Since $s \in S$ implies $P_{1, s}=1$, it follows easily using (2.1) that $S \subseteq \mathscr{D}$. Notice also that $a(z)=0$ if and only if $z=1$. For an irreducible $\mathbb{Q} W$-module, $E$, let $\widetilde{E}$ denote the lift to an $\mathscr{H}$-module as defined by Lusztig in $[9, \S 1]$, and let $a(E)$ be the highest power of $u$ dividing the generic degree of $\widetilde{E}$. We summarize the properties of $a$ and $\mathscr{D}$ that we will need in the following proposition. 


\section{(2.2) Proposition.}

(1) Let $x, y \in W$ and suppose that $x \leq_{L R} y$. Then $a(y) \leq a(x)$; in particular, $x \sim_{L R} y$ implies $a(x)=a(y)$.

(2) Let $\Gamma$ be a left cell in $W$. Then $|\Gamma \cap \mathscr{D}|=1$.

(3) Let $x, y \in W, x \leq_{L} y$ and $a(x)=a(y)$. Then $x \sim_{L} y$.

(4) Let $E$ be an irreducible $\mathbb{Q} W$-module and let $X$ be the two-sided cell corresponding to $E$. Then $a(E)=a(X)$, where $a(X)$ is defined to be the common value of $a(x)$ for $x \in X$.

Proof. These are all proved by Lusztig, [11, 5 and $\S 6],[12, \S 1]$, and [13, Proposition 3.3].

For the remainder of this paper, $\widetilde{E}$ will denote the $\mathscr{H}$-module described in $\S 1$ corresponding to the reflection representation of $W$. In order to determine the cells corresponding to $\widetilde{E}$, we need some preliminary lemmas.

(2.3) Lemma. For $x \in W, a(x)=1$ if and only if $x \sim_{L} s$ for some $s \in S$.

Proof. By $(2.2(1))$, if $x \sim_{L} s$, then $a(x)=a(s)=1$. Conversely, suppose that $a(x)=1$. We will prove the result using induction on $\ell(x)$. If $\ell(x)=0$ or $\ell(x)=1$, then the result clearly holds, so suppose $\ell(x)>1$. Choose $t \in S$ so that $t x<x$. Then $x \leq_{L} t x$, so by $(2.2(1)), a(t x) \leq a(x)=1$. If $a(t x)=0$, then $x=t \in S$, contradicting the assumption that $\ell(x)>1$. Hence $a(t x)=1=a(x)$, so by $(2.2(3)), x \sim_{L} t x$. By induction, $t x \sim_{L} s$ for some $s \in S$. This completes the proof of (2.3).

(2.4) Lemma. Let $s, s^{\prime} \in S$. Then $s \sim_{L R} s^{\prime}$.

Proof. If $s$ and $s^{\prime}$ are adjacent in the Coxeter graph of $(W, S)$, then it follows from the definition of $\leq_{L}$ and $\leq_{R}$ (see [8]) that $s \sim_{R} s s^{\prime} \sim_{L} s^{\prime}$, so $s \sim_{L R} s^{\prime}$. If $s$ and $s^{\prime}$ are not adjacent, then they are connected by a path (recall that ( $W, S)$ is indecomposable), and it follows from the transitivity of $\sim_{L R}$ that $S \sim_{L R} s^{\prime}$. This completes the proof of (2.4).

Now let $\Gamma$ be a left cell with $a(\Gamma)=1$. Then by (2.3) and (2.2(2)) there is a unique $s \in S$ so that $s \in \Gamma$. Denote $\Gamma$ by $\Gamma_{s}$. Let $X$ be a two-sided cell with $a(X)=1$. Then by (2.4) and (2.2), $X=\bigcup_{s \in S} \Gamma_{s}$, and this is the decomposition of $X$ into left cells.

(2.5) Theorem. Let $X$ be a two-sided cell of $W$, then the following are equivalent:

(1) $X$ is the two-sided cell corresponding to the reflection representation of $W$,

(2) $a(X)=1$,

(3) $X=\bigcup_{s \in S} \Gamma_{s}$, where for $s \in S, \Gamma_{s}$ is the left cell containing $s$, and

(4) $X=\{w \in W \mid w \neq 1$ and $w$ has a unique reduced expression $\}$.

Moreover, if $X$ satisfies (1)-(4) and $Y$ is another two-sided cell with $X \leq_{L R} Y$, then either $Y=X$ or $Y=\{1\}$. 
Proof. Let $X$ be the two-sided cell of $W$ corresponding to the reflection representation of $W$. From the formulas for the generic degree of $\widetilde{E}$ given in [3], we see that $a(\widetilde{E})=1$, so by $(2.2(4)),(1)$ is equivalent to (2).

By the remarks preceding the statement of the theorem, (2) implies (3).

Now suppose that $X$ is the two-sided cell of (3) and that $x \in X$. We will show by induction on $\ell(x)$ that $x$ has a unique reduced expression, and hence that (3) implies (4). If $\ell(x)=1$, then the result is clear. Suppose that $l(x)>1$ and choose $s \in S$ so that $s x<x$. The argument used in the proof of (2.3) shows that $s x \in X$ since $x \neq 1$, so by induction, $s x$ has a unique reduced expression. Since $x \in X$, it follows from (3) and the fact that $\mathscr{L}(\cdot)$ is constant on right cells (see [6]), that $\mathscr{L}(x)=\{s\}$ for some $s \in S$. Therefore, every reduced expression for $x$ begins with $s$, and so $x$ has a unique reduced expression.

Conversely, suppose $x \in W$ has a unique reduced expression and $x \neq 1$. We will show by induction on $\ell(x)$ that $x \in \Gamma_{t}$ for some $t \in S$. This is clear if $\ell(x)=1$. Suppose that $\ell(x)>1$ and choose $s \in S$ with $s x<x$. Since $x$ has a unique reduced expression, $\mathscr{L}(x)=\{s\}$ and $s x$ has a unique reduced expression. By induction, $s x \in \Gamma_{t}$ for some $t \in S$. Furthermore, $\mathscr{L}(s x) \cap \mathscr{L}(x)=\varnothing$ and so it follows from the definition of $\leq_{L}$ that $x \sim_{L} s x$. Hence $x \in \Gamma_{t}$.

It follows from the preceding paragraph and (2.2(1)) that if $x \in W$ has a unique reduced expression and $x \neq 1$, then $a(x)=1$, so (4) implies (2).

Finally, suppose that $X$ satisfies the equivalent conditions (1)-(4), and that $Y$ is a two-sided cell with $X \leq_{L R} Y$. Then by (2.2(1)), $a(Y) \leq a(X)$, so $a(Y) \in\{0,1\}$ and the result follows using the fact that $X$ is the unique twosided cell with $a(X)=1$. This completes the proof of (2.5).

For the rest of this paper, $X$ will denote the two-sided cell of (2.5).

We now turn to computing the matrix entries of $\widetilde{E}$. For $x \in W$ and $s, t \in S$, recall that $\pi(x, s, t) \in A$ is defined by

$$
T_{x} d_{s}=\sum_{t \in S} \pi(x, s, t) d_{t}
$$

Since $C_{s}=-u^{1 / 2} T_{1}+u^{-1 / 2} T_{s}$ for $s \in S$, it follows from (1.6) that

$$
C_{s} d_{t}= \begin{cases}-\left(u^{1 / 2}+u^{-1 / 2}\right) d_{s} & \text { if } s=t, \\ -\left\langle\alpha_{t}, \alpha_{s}\right\rangle d_{s} & \text { if } s \neq t .\end{cases}
$$

Define $k(w, s, t) \in A$ by

$$
C_{w} d_{s}=\sum_{t \in S} k(w, s, t) d_{t} .
$$

By [6, Theorem (3.1)], for $x \in W$,

$$
T_{x}=\sum_{w \in W} u_{x} u_{w}^{-1 / 2} \overline{Q_{u, x}} C_{w}
$$


where $Q_{w, x}=P_{w_{0} x, w_{0} w}$ and $\overline{Q_{x, w}\left(u^{1 / 2}\right)}=Q_{x, w}\left(u^{-1 / 2}\right)$. Hence,

$$
\pi(x, s, t)=\sum_{w \in W} u_{x} u_{w}^{-1 / 2} \overline{Q_{w, x}} k(w, s, t) .
$$

Fix $s, t \in S$ and recall that $\Gamma_{s}$ is the left cell containing $s$. Let $\Delta_{s, t}=$ $\Gamma_{s} \cap \Gamma_{t}^{-1}$. Notice that $X=\bigcup_{s, t} \Delta_{s, t}$. For $x \in W$, define

$$
c(x, s)= \begin{cases}1 & \text { if } x s<x \\ 0 & \text { if } x s>x .\end{cases}
$$

It will be shown below that when $w \in \Delta_{s, t}, k(w, s, t)$ has the form

$$
k(w, s, t)=-n_{w}\left(u^{1 / 2}+u^{-1 / 2}\right)
$$

where $n_{w} \in \mathbb{N}-\{0\}$.

(2.8) Theorem. For $x \in W$ and $s, t \in S$,

$$
\pi(x, s, t)=u_{x}\left[\overline{\sum_{w \in \Delta_{s, t}} n_{w} u_{w s}^{1 / 2} u^{c(x, s)}\left(Q_{w, x s}-Q_{w, x}\right)}\right] .
$$

The proof of (2.8) requires several preparatory lemmas. The strategy is to express the $k(y, s, t)$ for $y \in W$ in terms of the $n_{w}$ where $w \in \Delta_{s, t}$, and then to use the recursion relation (2.10) for the $Q_{w, x}$ 's to derive (2.8) from (2.7).

(2.9) Lemma. Suppose $x$ and $w$ in $W$ are such that $x<w, x \neq t w$ for all $t \in S$, and $\mu(x, w) \neq 0$. Then $\mathscr{L}(w) \subseteq \mathscr{L}(x)$.

Proof. Let $s \in \mathscr{L}(w)$ and just suppose $s x>x$. Then by $[6,(2.3 \mathrm{e})], \mu(x, w) \neq$ 0 if and only if $x=s w$, a contradiction, so $s x<x$. This proves (2.9).

For $w \in W$ with $\mathscr{R}(w)=\{s\}$, put $\mathscr{A}(w)=\{t \in S \mid \ell(w t s)=\ell(w)+2\}$.

(2.10) Lemma. Let $x, w \in W$, and suppose that $\mathscr{R}(w)=\{s\}$, then

$$
Q_{w s, x}=u^{c(x, s)} Q_{w, x s}+u^{1-c(x, s)} Q_{w, x}-u \sum_{t \in \mathscr{N}(w)} Q_{w t, x} .
$$

Proof. This is the recursion formula [6, (2.2c)] combined with (2.9). We will omit the details of the proof.

(2.11) Lemma. Let $x \in W$ and $t \in S$ with $t x<x$. Then $k\left(x, s, t^{\prime}\right)=0$ for all $t^{\prime} \in S$ with $t^{\prime} \neq t$.

Proof. This follows by comparing the coefficient of $d_{t^{\prime}}$ in $\left(C_{t} C_{x}\right) d_{s}$ and $C_{t}\left(C_{x} d_{s}\right)$. We will omit further details of the proof.

(2.12) Lemma. Let $w \in W$. Then

(1) $\overline{k(w, s, t)}=k(w, s, t)$, and

(2) the degree in $u^{1 / 2}$ of $k(w, s, t)$ is at most 1 . 
Proof. The proof is by induction on $\ell(w)$. If $\ell(w)=0$, then $w=1$ and $k(1, s, t)=\delta_{s, t}$, so (1) and (2) are true.

If $\ell(w)>0$, choose $t \in S$ with $t w<w$; then using (2.1) and comparing the coefficient of $d_{t}$ in $\left(C_{t} C_{t w}\right) d_{s}$ and $C_{t}\left(C_{t w} d_{s}\right)$ gives

$$
\begin{aligned}
k(w, s, t)+\sum_{\substack{x<t w \\
t x<x}} \mu(x, t w) k(x, s, t) & \\
& =-\left(u^{1 / 2}+u^{-1 / 2}\right) k(t w, s, t)-\sum_{t^{\prime} \neq t}\left\langle\alpha_{t^{\prime}}, \alpha_{t}\right\rangle k\left(t w, s, t^{\prime}\right) .
\end{aligned}
$$

Now (1) follows easily by induction.

If $\ell(w)=1$, then (2) follows from (2.6). Suppose $\ell(w)>1$ and $t_{0} \in S$ with $t_{0} t w<t w$. By (2.11), if $t^{\prime} \in S$ and $t^{\prime} \neq t_{0}$, then $k\left(t w, s, t^{\prime}\right)=0$. Since $t_{0} \neq t$, we have $k(t w, s, t)=0$, and so (2) follows from (2.13). This completes the proof of (2.12).

(2.14) Lemma. For $x \in W$ and $s, t \in S, k(x, s, t) \neq 0$ implies $x \in X \cup\{1\}$. Proof. Let $\phi: K \otimes_{A} \widetilde{E} \rightarrow K \otimes_{A} \mathscr{M}_{X}$ be a $K \otimes_{A} \mathscr{H}$-linear injection and put $d_{t}^{\prime}=\phi\left(d_{t}\right)$ for $t \in S$. Then for $x \in W$ and $s \in S$,

$$
C_{x} d_{s}^{\prime}=\sum_{t \in S} k(x, s, t) d_{t}^{\prime} .
$$

Write $d_{t}^{\prime}=\sum_{y \in X} \alpha_{t, y} c_{y}$ with $\alpha_{t, y} \in K$. Then

$$
C_{x} d_{s}^{\prime}=\sum_{y \in X}\left(\sum_{t \in S} k(x, s, t) \alpha_{t, y}\right) c_{y}
$$

and

$$
C_{x} d_{s}^{\prime}=\sum_{y \in X}\left(\sum_{z \in X} \alpha_{s, z} h_{x, z, y}\right) c_{y} .
$$

Comparing coefficients in (2.15) and (2.16) yields that for $y \in X$,

$$
\sum_{t \in S} k(x, s, t) \alpha_{t, y}=\sum_{z \in X} \alpha_{s, z} h_{x, z, y} \text {. }
$$

Since the $d_{t}^{\prime}$ are $K$-linearly independent, it follows that the equations (2.17) can be solved for the $k(x, s, t)$ 's. Therefore, each $k(x, s, t)$ is a $K$-linear combination of the $h_{x, z, y}$, where $y, z \in X$. Hence $k(x, s, t) \neq 0$ implies $h_{x, z, y} \neq 0$ for some $y, z \in X$. It follows from the definition of $\leq_{R}$ that $h_{x, z, y} \neq 0$ implies $y \leq_{R} x$. Hence $y \leq_{L R} x$, and since $y \in X$ it follows from (2.5) that $x \in X \cup\{1\}$. This completes the proof of (2.14).

(2.18) Lemma. Let $x \in X$ and suppose that $k(x, s, t) \neq 0$ and $\mathscr{R}(x)=\left\{s_{1}\right\}$. Then $\mathscr{L}(x)=\{t\}$, and either $s=s_{1}$ or $\left|s s_{1}\right|>2$. Moreover, if $\left|s s_{1}\right|>2$, then $k(x, s, t)$ has degree 0 in $u^{1 / 2}$.

Proof. Suppose $x \in X$ and $k(x, s, t) \neq 0$. Then $|\mathscr{L}(x)|=|\mathscr{R}(x)|=1$. It follows from (2.11) that $t \in \mathscr{L}(x)$, so $\mathscr{L}(x)=\{t\}$. 
Suppose $s \neq s_{1}$. Then comparing the coefficient of $d_{t}$ in $\left(C_{x} C_{s_{1}}\right) d_{s}$ and $C_{x}\left(C_{s_{1}} d_{s}\right)$ yields

$$
\left(u^{1 / 2}+u^{-1 / 2}\right) k(x, s, t)=\left\langle\alpha_{s}, \alpha_{s_{1}}\right\rangle k\left(x, s_{1}, t\right) .
$$

Hence $k(x, s, t) \neq 0$ implies that $\left\langle\alpha_{s}, \alpha_{s_{1}}\right\rangle \neq 0$, so $\left|s s_{1}\right|>2$. By $(2.12(2))$, $k\left(x, s_{1}, t\right)$ has degree at most 1 in $u^{1 / 2}$, so $k(x, s, t)$ has degree 0 in $u^{1 / 2}$. This completes the proof of (2.18).

(2.19) Lemma. Let $w \in \Gamma_{s}$ and let $t \in \mathscr{A}(w)$. Then $w t \in X$.

Proof. We will show, using induction on $\ell(w)$, that $w t$ has a unique reduced expression, so by (2.5(4)), $w t \in X$. If $\ell(w)=1$, then the result is obvious. Suppose that $\ell(w)>1$. Because $w$ has a unique reduced expression, it suffices to show that $\mathscr{R}(w t)=\{t\}$. Let $t^{\prime} \in \mathscr{R}(w t)$ and just suppose that $t^{\prime} \neq t$. Fix a reduced expression for $w, w=s_{1} \cdots s_{r}$, where $s_{i} \in S$ for $1 \leq i \leq r$. By the Exchange Property and our assumption that $t \neq t^{\prime}, w t t^{\prime}=s_{1} \cdots \widehat{s}_{i} \cdots s_{r} t$ for some $i$, where ${ }^{-}$means delete. There are three cases.

First, suppose that $i=1$ and put $w^{\prime}=s_{2} \cdots s_{r}$. Then $w^{\prime} \in X$ by (2.5(4)), and it is easily checked that $t \in \mathscr{A}\left(w^{\prime}\right)$. Hence, by induction, $w^{\prime} t \in X$. Since $\mathscr{L}(w t)=\left\{s_{1}\right\}$ it follows that $w t$ has a unique reduced expression, so $w t \in X$. But this implies that $t=t^{\prime}$, a contradiction. Thus we may assume that $i>1$.

Next, suppose that $1<i<r$ and put $w^{\prime}=s_{i} \cdots s_{r}$. Then $w^{\prime} \in X$ by (2.5(4)), and it is easily checked that $t \in \mathscr{A}\left(w^{\prime}\right)$. Hence, by induction, $w^{\prime} t \in$ $X$. Thus $t^{\prime}=t$, and again we obtain a contradiction.

Finally, suppose that $i=r$. Then $w t t^{\prime}=w s_{r} t$, hence $t s_{r} t=t^{\prime}$. By assumption, $t \in \mathscr{A}(w)$, so $|t s|>2$. Since $s=s_{r}$, it follows that $\ell\left(t s_{r} t\right)=3$, contradicting our previous conclusion that $t s_{r} t=t^{\prime}$.

Since we have arrived at a contradiction in each of the preceding cases, we must have that $t=t^{\prime}$. Hence $w t \in X$, as desired. This completes the proof of (2.19).

For $s, t \in S$, define $\overline{\Delta_{s, t}}$ to be $\left\{w t \mid w \in \Delta_{s, t}\right.$ and $\left.t \in \mathscr{A}(w)\right\}$.

(2.20) Lemma. Let $w \in W$. Then $k(w, s, t) \neq 0$ implies $w \in \Delta_{s, t} \cup \overline{\Delta_{s, t}} \cup \Delta_{s, t} s \cup$ $\{1\}$.

Proof. Just suppose that $k(w, s, t) \neq 0$ and $w \notin \Delta_{s, t} \cup \overline{\Delta_{s, t}} \cup \Delta_{s, t} \cup\{1\}$. By (2.14), $w \in X$, and by (2.18), if $\mathscr{R}(w)=\left\{s^{\prime}\right\}$, then either $s=s^{\prime}$ or $\left|s s^{\prime}\right|>2$. If $s=s^{\prime}$, then it follows from (2.18) that $w \in \Delta_{s, t}$, a contradiction. Hence $\left|s s^{\prime}\right|>2$.

If $s \in \mathscr{A}(w)$, then by (2.19) $w s \in X$, so $w s \in \Delta_{s, t}$, contradicting the assumption that $w \notin \Delta_{s, t} s$. Hence $s \notin \mathscr{A}(w)$, so $w s s^{\prime}<w s$.

If $w s^{\prime} s<w s^{\prime}$, then $\ell\left(w s^{\prime}\right) \geq 1$. Put $w s^{\prime} s=x$. If $x=1$, then by (2.18), $k(w, s, t) \neq 0$ implies $\mathscr{L}(w)=\{t\}$, so $s=t$. Since $\left|s s^{\prime}\right|>2$ we must have that $s s^{\prime} \in \overline{\Delta_{s, t}}$, a contradiction. Thus $x \neq 1$. It follows from (2.5) that 
$x \in X$ and so $\mathscr{L}(x)=\{t\}$. Hence $x s=w s^{\prime} \in \Delta_{s, t}$. Since $s^{\prime} \in \mathscr{A}\left(w s^{\prime}\right)$, it follows that $w=w s^{\prime} s^{\prime} \in \overline{\Delta_{s, t}}$, a contradiction. We may therefore conclude that $w s^{\prime} s>w s^{\prime}$.

By two applications of Property Z (Deodhar [4]) we have:

(1) $w s s^{\prime}<w s$ implies that $w s^{\prime}<w s s^{\prime}<w s$ and

(2) $w s^{\prime}<w s^{\prime} s$ implies that $w s^{\prime}<w s^{\prime} s<w s$.

Since $w s^{\prime}<w<w s$ and the open interval $\left(w s^{\prime}, w s\right)$ in $W$ has exactly two elements (Björner [1, Proposition 1]), we must have that either $s=s^{\prime}$ or $\left|s s^{\prime}\right|=2$. In any case, we obtain a contradiction. This completes the proof of (2.20).

We next prove the converse of (2.20) in which we complete the computation of the $k(w, s, t)$ for $w \in W$ and $s, t \in S$. Let $y \in \overline{\Delta_{s, t}}$. Then $y=w t^{\prime}$ for some unique $w \in \Delta_{s, t}$ and $t^{\prime} \in \mathscr{A}(w)$. Define $\bar{y}$ to be this $w$. Notice that $y \in\left(\Delta_{s, t}\right) s$ implies $y s \in \Delta_{s, t}$.

\section{(2.21) Proposition.}

(1) Let $w \in \Delta_{s, t}$. Then $k(w, s, t)=-n_{w}\left(u^{1 / 2}+u^{-1 / 2}\right)$, where $n_{w}$ is a positive integer. Hence $n_{w}$ is defined whenever $w \in \Delta_{s, t}$.

(2) Let $y \in \overline{\Delta_{s, t}}-\left(\Delta_{s, t}\right) s$. Then $k(y, s, t)=n_{\bar{y}}$.

(3) Let $y \in\left(\Delta_{s, t}\right) s-\overline{\Delta_{s, t}}$. Then $k(y, s, t)=n_{y s}$.

(4) Let $y \in\left(\Delta_{s, t}\right) s \cap \overline{\Delta_{s, t}}$. Then $k(y, s, t)=n_{y s}+n_{\bar{y}}$.

Proof. (1) We will prove this statement for all $s, t \in S$ simultaneously using induction on $\ell(w)$. If $\ell(w)=1$ and $w \in \Delta_{s, t}$, then $w=s=t$ and $k(s, s, s)=$ $-\left(u^{1 / 2}+u^{-1 / 2}\right)$, so (1) holds. Next suppose that $\ell(w)=2$ and $w \in \Delta_{s, t}$, then $w=t s$. It follows from (2.1) that $C_{t s}=C_{t} C_{s}$, so

$$
k(t s, s, t)=\left\langle\alpha_{s}, \alpha_{t}\right\rangle\left(u^{1 / 2}+u^{-1 / 2}\right),
$$

and so (1) holds in this case.

Now suppose that $\ell(w)>2$ and that the result holds whenever $s, t \in S$ and $x \in \Delta_{s, t}$ with $\ell(x)<\ell(w)$. Let $w \in \Delta_{s, t}$. Then since $\ell(w)>2$, it follows from $(2.5(4))$ that $t w \in X$.

Suppose $\mathscr{L}(t w)=\left\{t_{1}\right\}$. Then comparing the coefficient of $d_{t}$ in $\left(C_{t} C_{t w}\right) d_{s}$ and $C_{t}\left(C_{t w} d_{s}\right)$, and using (2.1) yields

$$
\begin{aligned}
k(w, s, t)+\sum_{\substack{x<t w \\
t x<x}} \mu(x, t w) k(x, s, t) & \\
= & -\left(u^{1 / 2}+u^{-1 / 2}\right) k(t w, s, t)-\sum_{t_{2} \neq t}\left\langle\alpha_{t_{2}}, \alpha_{t}\right\rangle k\left(t w, s, t_{2}\right) .
\end{aligned}
$$

Suppose $x \in W$ with $\mu(x, t w) k(x, s, t) \neq 0, x<t w$ and $t x<x$. Then $x \neq 1$ and so by (2.14), $x \in X$. If $x \neq s^{\prime} t w$ for all $s^{\prime} \in S$, then by (2.9), $\mathscr{L}(t w) \subseteq \mathscr{L}(x)=\{t\}$. But then $t_{1}=t$, which is impossible, since $w \in X$ 
and $t_{1} t w<t w<w$. Hence $x=t_{1} t w$. Also, if $t_{2} \in S$ and $t_{2} \neq t_{1}$, then by (2.18), $k\left(t w, s, t_{2}\right)=0$. Therefore, we may rewrite $(2.22)$ as

$$
k(w, s, t)+k\left(t_{1} t w, s, t\right)=-\left\langle\alpha_{t_{1}}, \alpha_{t}\right\rangle k\left(t w, s, t_{1}\right) .
$$

If $t t_{1} t w>t_{1} t w$, then $k\left(t_{1} t w, s, t\right)=0$ by (2.18). Since $t w \in \Delta_{s, t_{1}}$, (1) follows by induction from (2.23).

If $t t_{1} t w<t_{1} t w$, then $\left|t t_{1}\right|>3$, since $w$ has a unique reduced expression. Repeating the preceding argument with $k(w, s, t)$ replaced by $k\left(t w, s, t_{1}\right)$ yields:

$$
k(w, s, t)=\left\langle\alpha_{t_{1}}, \alpha_{t}\right\rangle k\left(t t_{1} t w, s, t_{1}\right)+\left(\left\langle\alpha_{t_{1}}, \alpha_{t}\right\rangle\left\langle\alpha_{t}, \alpha_{t_{1}}\right\rangle-1\right) k\left(t_{1} t w, s, t\right) .
$$

Suppose $\left|t t_{1}\right|=4$. If $w=t t_{1} t$, then an easy computation shows that (1) holds. Otherwise, $\ell(w)>4$, and so by (2.18) and (2.5(4)), $k\left(t t_{1} t w, s, t_{1}\right)=0$, and so (1) follows by induction from (2.24).

If $\left|t t_{1}\right|=6$, then $W$ is of type $G_{2}$ and (1) is easily proved by explicitly computing the $k(w, s, t)$ (see $\S 4)$.

(2) Suppose $y \in \overline{\Delta_{s, t}}-\left(\Delta_{s, t}\right) s$. Let $y=w t^{\prime}$ where $w \in \Delta_{s, t}$ and $t^{\prime} \in$ $\mathscr{A}(w)$, so $w<w t^{\prime}<w t^{\prime} s$. If $w t^{\prime} s \in X$, then $w t^{\prime} s \in \Delta_{s, t}$, so $y \in\left(\Delta_{s, t}\right) s$, a contradiction. Hence $w t^{\prime} s \notin X$, so by (2.14), $k\left(w t^{\prime} s, s_{1}, t_{1}\right)=0$ for all $s_{1}, t_{1} \in S$. Comparing the coefficient of $d_{t}$ in $\left(C_{w t^{\prime}} C_{s}\right) d_{s}$ and $C_{w t^{\prime}}\left(C_{s} d_{s}\right)$, and using (2.1) yields

$$
k\left(w t^{\prime} s, s, t\right)+\sum_{\substack{x<w t^{\prime} \\ x s<x}} \mu\left(x, w t^{\prime}\right) k(x, s, t)=-\left(u^{1 / 2}+u^{-1 / 2}\right) k\left(w t^{\prime}, s, t\right) .
$$

The argument used to prove (1) gives that when $\mu\left(x, w t^{\prime}\right) k(x, s, t) \neq 0, x<w t^{\prime}$ and $x s<x$, we must have that $x=w$. Thus (2.25) can be rewritten as

$$
k(w, s, t)=-\left(u^{1 / 2}+u^{-1 / 2}\right) k\left(w t^{\prime}, s, t\right) .
$$

This proves $(2.21(2))$.

(3) Suppose that $y \in\left(\Delta_{s, t}\right) s-\overline{\Delta_{s, t}}$. Notice that $y<y s$.

First suppose that $y=1$. Then $s \in \Delta_{s, t}$, so $s=t$ and $k(y, s, s)=1$. It follows from (2.6) that $n_{s}=1$, so (3) holds.

Next, suppose that $y \in S$. Then $y s \in \Delta_{s, t}$ implies that $y=t$ and $|s t|>2$. It follows from (2.1) that $C_{t} C_{s}=C_{t s}$. Thus, (3) follows from (2.6).

Finally, suppose that $\ell(y)>1$. Let $\mathscr{R}(y)=\left\{s^{\prime}\right\}$, so $\left|s s^{\prime}\right|>2$, and $y s^{\prime} \in X$. If $y s^{\prime} \in \Delta_{s, t}$, then $y=y s^{\prime} s^{\prime} \in \overline{\Delta_{s, t}}$, a contradiction. Hence $y s^{\prime} \notin \Delta_{s, t}$. Now $\ell(y) \geq 2$, so $t y s^{\prime}<y s^{\prime}$; so since $y s^{\prime} \notin \Delta_{s, t}$, but $y s^{\prime} \in X$, we must have that $y s^{\prime} s>y s^{\prime}$.

Comparing the coefficient of $d_{t}$ in $\left(C_{y} C_{s}\right) d_{s}$ and $C_{y}\left(C_{s} d_{s}\right)$, using (2.1), and repeating the argument used in (1), yields

$$
-\left(u^{1 / 2}+u^{-1 / 2}\right) k(y, s, t)=k(y s, s, t)+k\left(y s^{\prime}, s, t\right) .
$$


Just suppose that $k\left(y s^{\prime}, s, t\right) \neq 0$. Then by (2.14), $y s^{\prime} \in X$. By (2.18), if $\mathscr{R}\left(y s^{\prime}\right)=\left\{s^{\prime \prime}\right\}$ then either $s^{\prime \prime}=s$ or $\left|s^{\prime \prime} s\right|>2$. By the preceding paragraph, $s^{\prime \prime} \neq s$, so $\left|s^{\prime \prime} s\right|>2$. Since $y s^{\prime} s^{\prime \prime}<y s^{\prime}<y<y s$ and $y s \in X$, it follows that $\ell\left(s^{\prime \prime} s^{\prime} s\right)=3$ and $s^{\prime \prime} s^{\prime} s \in X$. But then $\left\{s^{\prime \prime}, s^{\prime}, s\right\}$ corresponds to a cycle in the Coxeter graph of $W$, contradicting the indecomposability of $W$. Therefore $k\left(y s^{\prime}, s, t\right)=0$, and so by $(2.26)$ we conclude that $-\left(u^{1 / 2}+u^{-1 / 2}\right) k(y, s, t)=$ $k(y s, s, t)$.

(4) Suppose that $y \in\left(\Delta_{s, t}\right) s \cap \overline{\Delta_{s, t}}$. Since $y \in\left(\Delta_{s, t}\right) s, y<y s$, and $y \in \overline{\Delta_{s, t}}$, we must have $\ell(y)>2$. Let $\mathscr{R}(y)=\left\{s^{\prime}\right\}$. Then $y s \in X$ implies $\left|s^{\prime} s\right|>2$. Write $y=w t^{\prime}$ where $w \in \Delta_{s, t}$ and $t^{\prime} \in \mathscr{A}(w)$. Then $y t^{\prime}<y$, so $t^{\prime}=s^{\prime}$ and $w=y s^{\prime} \in \Delta_{s, t}$. Comparing the coefficient of $d_{t}$ in $\left(C_{y} C_{s}\right) d_{s}$ and $C_{y}\left(C_{s} d_{s}\right)$, and using (2.1) and the argument in (1), gives

$$
-\left(u^{1 / 2}+u^{-1 / 2}\right) k(y, s, t)=k(y s, s, t)+k\left(y s^{\prime}, s, t\right),
$$

and (4) follows easily.

We can now prove (2.8). Let $x \in W$ and $s, t \in S$. Using (2.7) and (2.12(1)), we see that

$$
\pi(x, s, t)=u_{x}\left[\overline{\sum_{w} u_{w}^{1 / 2} Q_{w, x} k(w, s, t)}\right],
$$

so it suffices to prove

$$
\sum_{w \in W} u_{w}^{1 / 2} Q_{w, x} k(w, s, t)=\sum_{w \in \Delta_{s, t}} n_{w} u_{w s}^{1 / 2} u^{c(x, s)}\left(Q_{w, x s}-Q_{w, x}\right) .
$$

We start with the right-hand side (r.h.s.) of (2.27) and the observation that

$$
-u^{c(x, s)}=u^{1-c(x, s)}-1-u .
$$

Then using (2.10), (2.20), and (2.21), we have

$$
\begin{aligned}
\text { r.h.s. }= & \sum_{w \in \Delta_{s, t}} n_{w} u_{w s}^{1 / 2}\left(u^{c(x, s)} Q_{w, x s}+u^{1-c(x, s)} Q_{w, x}-(1+u) Q_{w, x}\right) \\
= & \sum_{u \in \Delta_{s, t}} n_{w} u_{w s}^{1 / 2}\left(Q_{w s, x}+\sum_{t^{\prime} \in \mathscr{S}(w)} u Q_{w t^{\prime}, x}-(1+u) Q_{w, x}\right) \\
= & \sum_{y \in\left(\Delta_{s, t}\right) s} n_{y s} u_{y}^{1 / 2} Q_{y, x}+\sum_{w \in \Delta_{s, t}} \sum_{t^{\prime} \in \mathscr{A}(w)} n_{w} u_{w t^{\prime}}^{1 / 2} Q_{w t^{\prime}, x} \\
& -\sum_{w \in \Delta_{s, t}} n_{w}\left(u^{1 / 2}+u^{-1 / 2}\right) u_{w}^{1 / 2} Q_{w, x} \\
= & \sum_{w \in W} u_{w}^{1 / 2} Q_{w, x} k(w, s, t) .
\end{aligned}
$$

This completes the proof of $(2.8)$ 


\section{WeYl GROUPS OF TYPE $\mathrm{A}_{l-1}$ AND $\mathrm{B}_{l}$}

In this section we collect the results about Weyl groups of type $\mathrm{A}_{l-1}$ and

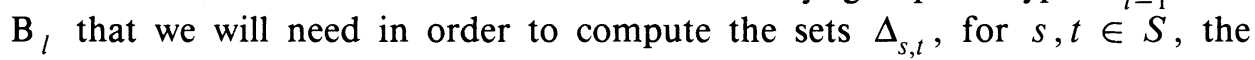
positive integers $n_{w}$, and the polynomials $Q_{w, x},\left(w \in \Delta_{s, t}, x \in W\right)$, of (2.8). In particular we will describe the minimal double coset representatives for all pairs of maximal parabolic subgroups.

Given a positive integer, $n$, let $[n]$ denote the set $\{1, \ldots, n\}$.

Fix an integer, $l$, with $l \geq 2$.

Let $B$ be the group of all signed permutations of $[l]$. We will identify elements of $B$ with their images. Thus, we will denote the permutation

$$
\left(\begin{array}{cccc}
1 & 2 & \cdots & l \\
\pm i_{1} & \pm i_{2} & \cdots & \pm i_{l}
\end{array}\right)
$$

(where $\left.\left\{i_{1}, \ldots, i_{l}\right\}=[l]\right)$ by the row vector $\left( \pm i_{1}, \ldots, \pm i_{l}\right)$. For $1 \leq i \leq l$ define elements, $s_{i}$, of $B$ by

$$
\begin{aligned}
s_{1} & =(2,1,3,4, \ldots, l) \\
s_{2} & =(1,3,2,4, \ldots, l) \\
& \vdots \\
s_{l-1} & =(1, \ldots, l-2, l, l-1) \\
s_{l} & =(1, \ldots, l-2, l-1,-l) .
\end{aligned}
$$

Let $S=\left\{s_{1}, \ldots, s_{l}\right\}$. Then $(B, S)$ is a Coxeter system of type $\mathbf{B}_{l}$.

For $w \in B$, define $N(w)$ to be $\{j \in[l] \mid w(j)<0\}$. For example, if $w=(-3,2,-1)$, then $N(w)=\{1,3\}$. Let $A=\{w \in B \mid N(w)=\varnothing\}$. Then $(A, A \cap S)$ is a Coxeter system of type $\mathrm{A}_{l-1}$. Since the ring $A$, of $\S 1$ and $\S 2$, does not appear in this section, the notation should not cause any confusion.

\section{(3.1) Proposition.}

(1) Let $1 \leq i \leq l-1$ and let $w \in B$. Then $w s_{i}<w$ if and only if: $i, i+1 \notin N(w)$ and $w(i)>w(i+1)$, or $i, i+1 \in N(w)$ and $|w(i)|<$ $|w(i+1)|$, or $i \in N(w)$ and $i+1 \notin N(w)$.

(2) Let $w \in B$. Then $w s_{l}<w$ if and only if $w(l)<0$.

Proof. This is just a reformulation of the well-known result: Let $W$ be a Weyl group with root system $\Phi, \Pi$ a base of $\Phi, \alpha \in \Pi$, and $s$ the reflection corresponding to $\alpha$. Then $w s<w$ if and only if $w(\alpha) \in \Phi^{-}$, where $\Phi^{-}$ is the set of negative roots determined by $\Pi$. We omit further details of the proof.

For $1 \leq j \leq l$, define $B_{j}$ to be the subgroup of $B$ generated by

$$
\left\{s_{i} \mid 1 \leq i \leq l, i \neq j\right\} \text {. }
$$

Define $A_{j}$ to be $A \cap B_{j}$. Notice that $A=A_{l}=B_{l}$, and if $j<l$, then $A_{j}$ is generated by

$$
\left\{s_{i} \mid 1 \leq i \leq l-1, i \neq j\right\} \text {. }
$$


Define $X_{j}$ to be $\left\{w \in B \mid w s_{i}>w \forall i \neq j\right\}$. Then $X_{j}$ is the set of minimal left coset representatives of $B_{j}$ in $B$. Let $w \in A$. Then $w s_{l}>w$. It follows from (3.1) that if $j<l$, then $X_{j} \cap A$ is the set of minimal left coset representatives of $A_{j}$ in $A$.

The sets $X_{j}$ and $X_{j} \cap A$ can be described as follows: Let $\alpha$ and $\beta$ be disjoint subsets of $[l]$; say $\alpha=\left\{p_{1}<\cdots<p_{a}\right\}, \beta=\left\{q_{1}<\cdots<q_{b}\right\}$, and $[l]-(\alpha \cup \beta)=\left\{r_{1}<\cdots<r_{c}\right\}$. Define $w_{\alpha, \beta}$ by

$$
w_{\alpha, \beta}=\left(p_{1}, \ldots, p_{a},-q_{b}, \ldots,-q_{1}, r_{1}, \ldots, r_{c}\right) .
$$

\section{(3.3) Proposition.}

(1) $X_{j}=\left\{w_{\alpha, \beta} \mid \alpha, \beta \subseteq[l], \alpha \cap \beta=\varnothing\right.$, and $\left.|\alpha \cup \beta|=j\right\}$.

(2) $X_{j} \cap A=\left\{w_{\alpha, \varnothing} \mid \alpha \subseteq[l]\right.$ and $\left.|\alpha|=j\right\}$.

Proof. Clearly, (1) implies (2), and (1) follows directly from the definition of $X_{j},(3.1)$, and the observation that

$$
\left|B: B_{j}\right|=2^{j}\left(\begin{array}{l}
l \\
j
\end{array}\right)=\mid\{(\alpha, \beta) \mid \alpha, \beta \subseteq[l], \alpha \cap \beta=\varnothing \text { and }|\alpha \cup \beta|=j\} \mid .
$$

This completes the proof of (3.3)

Now suppose $1 \leq j, k \leq l$, and define $X_{j, k}$ to be $X_{j}^{-1} \cap X_{k}$. Then $X_{j, k}$ is the set of minimal $\left(B_{j}, B_{k}\right)$-double coset representatives, and if $j, k<l$, then $X_{j, k} \cap A$ is the set of minimal $\left(A_{j}, A_{k}\right)$-double coset representatives. Our goal is to describe $X_{j, k}$ and $X_{j, k} \cap A$. We will need the following characterization of the subgroups $A_{j}$ and $B_{j}$.

(3.4) Proposition.

(1) Suppose $1 \leq j \leq l$. Then

$$
B_{j}=\operatorname{stab}_{B}([j])=\operatorname{stab}_{B}(\{-1, \ldots,-j\}) .
$$

(2) Suppose $1 \leq j \leq l-1$. Then

$$
A_{j}=\operatorname{stab}_{A}([j]) \text {. }
$$

Proof. Both (1) and (2) follow directly from the definitions. We will omit the details of the proof.

For $1 \leq j, k \leq l$, define $\nu_{j, k}: B \rightarrow \mathbb{N} \times \mathbb{N}$ by

$$
\nu_{j, k}(x)=(|[j] \cap x([k])|,|-[j] \cap x([k])|),
$$

where $-[j]=\{-1, \ldots,-j\}$.

For the rest of this section we will fix $j$ and $k$ and write $\nu$ for $\nu_{j, k}$. Notice that if $x \in A$, then $\nu(x)=(|[j] \cap x([k])|, 0)$. 


\section{(3.5) Proposition.}

(1) Let $x, y \in B$. Then $B_{j} x B_{k}=B_{j} y B_{k}$ if and only if $\nu(x)=\nu(y)$.

(2) Let $x, y \in A$ and $j, k<l$. Then $A_{j} x A_{k}=A_{j} y A_{k}$ if and only if $\nu(x)=\nu(y)$.

Proof. Suppose first that (1) has been proved. Then (2) is proved as follows: If $x, y \in A$ with $A_{j} x A_{k}=A_{j} y A_{k}$ then by (1), $\nu(x)=\nu(y)$. Conversely, if $\nu(x)=\nu(y)$, then by (1), $B_{j} x B_{k}=B_{j} y B_{k}$. Since each $B_{j}(1 \leq j \leq l-1)$ can be factored as $A_{j}$ times a normal elementary abelian 2-group, it is easily shown that $A_{j} x A_{k}=A_{j} y A_{k}$.

We now prove (1). Since $B_{j} x B_{k}=B_{k} x^{-1} B_{j}$ and $\nu_{j, k}(x)=\nu_{k, j}\left(x^{-1}\right)$, we will assume, without loss of generality, that $j \leq k$.

Let $x, y \in B$ and suppose that $B_{j} x B_{k}=B_{j} y B_{k}$. Let $w_{j} \in B_{j}$ and $w_{k} \in B_{k}$ with $w_{j} x w_{k}=y$. Then, using $(3.4(1))$, we have

$$
\begin{aligned}
{[j] \cap y([k]) } & =[j] \cap w_{j} x w_{k}([k]) \\
& =w_{j}^{-1}([j]) \cap x([k]) \\
& =[j] \cap x([k]) .
\end{aligned}
$$

Similarly,

$$
\{-1, \ldots,-j\} \cap y([k])=\{-1, \ldots,-j\} \cap x([k]),
$$

so $\nu(x)=\nu(y)$.

Finally, suppose that $x, y \in B$ and $\nu(x)=\nu(y)$. By the preceding paragraph, we may assume that $x, y \in X_{k}$. Let $x=w_{\alpha, \beta}$, where the labeling is as in (3.2), and suppose $\nu(x)=(m, n)$. Then, since we have assumed that $j \leq k$, it follows that $m+n \leq k$. An easy computation now gives

$$
x^{-1}([j])=\{1, \ldots, m\} \cup\{k+1, \ldots, k+j-m-n\} .
$$

In particular, $x^{-1}([j])$ depends only on $m$ and $n$, and not on $\alpha$ and $\beta$, so $x^{-1}([j])=y^{-1}([j])$. Therefore, by (3.4), $B_{j} x=B_{j} y$, and so $B_{j} x B_{k}=$ $B_{j} y B_{k}$. This completes the proof of (3.5).

For the remainder of this section we will assume that $j \leq k$. For nonnegative integers $a$ and $b$ with $j+k-l \leq a+b \leq j$, define $x_{a, b}^{j, k}$, in $B$ by

$$
\begin{aligned}
x_{a, b}^{j, k}=(1, \ldots, a, j+1, \ldots, j+k-a-b,-j, \ldots, & \\
& -j+b-1, a+1, \ldots, j-b, j+k-a-b+1, \ldots, l) .
\end{aligned}
$$

(3.7) Corollary.

(1) Let $j$ and $k$ be such that $1 \leq j \leq k \leq l$. Then

$$
X_{j, k}=\left\{x_{a, b}^{j, k} \mid j+k-l \leq a+b \leq j\right\} .
$$

(2) Let $j$ and $k$ be such that $1 \leq j \leq k \leq l-1$. Then

$$
X_{j, k} \cap A=\left\{x_{a, 0}^{j, k} \mid j+k-l \leq a \leq j\right\} .
$$


Proof. This follows directly from (3.1) and (3.5). Once again we omit the details.

\section{Determination of $X$}

Recall that $X$ is the two-sided cell corresponding to the reflection representation of $W$. In this section we will describe reduced expressions for all the elements in $X$ and compute the $n_{w}$ of $\S 2$ for each type of irreducible Weyl group. If the Coxeter graph of $W$ has no multiple bonds, this is accomplished in (4.2) and (4.3). The remaining cases ( $W$ of type $\mathrm{B}_{l}[l \geq 2], \mathrm{F}_{4}$ or $\mathrm{G}_{2}$ ) are dealt with individually. For $W$ of type $\mathrm{F}_{4}$ or $\mathrm{G}_{2}$, we merely state the results and omit the proofs (the proofs in the case in which $W$ is of type $\mathrm{F}_{4}$ are similar to the proofs we will give for the case in which $W$ is of type $\mathrm{B}_{l}$ ). Hence most of this section is devoted to describing $X$ and computing the $n_{w}$ when $W$ is of type $\mathrm{B}_{l}$.

Recall our assumption that $W$ is finite and indecomposable, so its Coxeter graph is a tree. Thus, given $s, t \in S$ (not necessarily distinct) there is a unique path in the Coxeter graph of $W$ from the node corresponding to $s$ to the node corresponding to $t$. Let $s_{1}, \ldots, s_{r}$ be the elements of $S$ corresponding to this path, where the labeling is such that $s_{1}=s, s_{r}=t, s_{i}$ and $s_{i+1}$ are adjacent in the Coxeter graph if $1 \leq i \leq r-1$, and $s_{i}, s_{j}$ are not adjacent if $|i-j|>1$. Define $x_{s, t}$ to be $s_{1} \cdots s_{r}$. Notice that $x_{s, s}=s$, and if $|s t|>2$ then $x_{s, t}=s t$. Let $X^{\prime}=\left\{x_{s, t} \mid s, t \in S\right\}$.

Suppose that $x_{s, t}=s_{1} \cdots s_{r} \in X^{\prime}$. It is easily shown that $\mathscr{L}\left(x_{s, t}\right)=\{s\}$ and that $\mathscr{R}\left(x_{s, t}\right)=\{t\}$, so $x_{s, t} \in \Delta_{s, t}$. Applying this result to each $s_{i} \cdots s_{r}$ $(1 \leq i<r)$, and using the definition of $\leq_{L}$ [8] we have

$$
s_{1} \cdots s_{r} \sim_{L} s_{2} \cdots s_{r} \sim_{L} \cdots \sim_{L} s_{r} .
$$

Therefore $X^{\prime} \subseteq X$.

(4.1) Lemma. Let $\left\{s_{1}, \ldots, s_{r}\right\}$ be a subset of $S$ and put $w=s_{1} \cdots s_{r}$. Then in $\mathscr{H}$ we have $C_{w}=C_{s_{1}} \cdots C_{s_{r}}$.

Proof. This follows easily using induction and (2.1). We omit the details.

(4.2) Proposition. Let $s, t \in S$ and put $w=x_{s, t}=s_{1} \cdots s_{r}$, where the notation is as above. Then if $\alpha_{i}$ is the simple root corresponding to $s_{i}$ we have,

$$
n_{w}=(-1)^{r+1} \prod_{i=1}^{r-1}\left\langle\alpha_{i+1}, \alpha_{i}\right\rangle .
$$

The empty product is interpreted to be 1. In particular, if the Coxeter graph determined by $\left\{s_{1}, \ldots, s_{r}\right\}$ has no multiple bonds, then $n_{w}=1$.

Proof. This follows easily by recursion using (4.1), (1.6), and the definition of $n_{w}$. We omit further details. 
(4.3) Proposition. $X=X^{\prime}$ if and only if the Coxeter graph of $W$ has no multiple bonds.

Proof. Suppose first that the Coxeter graph of $W$ has a multiple bond. Then there exists $s$ and $t$ in $S$ with $|s t|>3$. By (2.5(4)), sts $\in X \backslash X^{\prime}$. Conversely, suppose that the Coxeter graph of $W$ has no multiple bonds, and just suppose that $x \in X \backslash X^{\prime}$. Since $x \notin X^{\prime}$ and $x \in X$, there is an $s \in S$ that appears at least twice in the reduced expression for $x$. By (2.5(4)), if $t \in S, t x<x$, and $t x \neq 1$, then $t x$ has a unique reduced expression, so $t x \in X$. Hence, by multiplying $x$ on the left and right by elements of $S$, we may assume that $x$ has the form sws, where $s \in S, w \in X$ with no element of $S$ appearing more than once in the reduced expression for $w, l(x)=l(w)+2$, and $s \leq x$. Since $w$ has a unique reduced expression, and the elements of $S$ appearing in this expression are distinct, it must be that adjacent elements in this expression do not commute. Thus $w \in X^{\prime}$. If $l(w)=1$, say $w=t \in S$; then sts $\in X$, so $s t$ has order at least 4 , a contradiction. Hence we must have that $l(w)>$ 1. Let $w=x_{s_{1}, s_{r}}=s_{1} \cdots s_{r}$ with the notation of the paragraph preceding (4.1). Now, since sws $\in X, s$ and $s_{1}$ do not commute and $s$ and $s_{r}$ do not commute. Hence $\left\{s, s_{1}, \ldots, s_{r}\right\}$ determines a cycle in the Coxeter graph of $W$, a contradiction. Therefore we may conclude that $X=X^{\prime}$.

Now suppose that $W$ is of type $\mathrm{G}_{2}$. Let $S=\left\{s_{1}, s_{2}\right\}$, and write $\alpha_{i}$ for $\alpha_{s_{i}}(i=1,2)$. Assume that $\alpha_{1}$ is a short root. It is easily shown that $X=$ $W \backslash\left\{1, w_{0}\right\}$. In what follows we will denote the simple reflection, $s_{i}$, simply by $i(i=1,2)$. Thus, 121 represents the element $s_{1} s_{2} s_{1}$ of $W$. Then we have

$$
X \backslash X^{\prime}=\{121,1212,12121,212,2121,21212\}
$$

and

$$
\begin{array}{lll}
n_{121}=2, & n_{12121}=1, & n_{2121}=1, \\
n_{1212}=3, & n_{212}=2, & n_{21212}=1 .
\end{array}
$$

Next suppose that $W$ is of type $\mathrm{F}_{4}$. Let $S=\left\{s_{1}, s_{2}, s_{3}, s_{4}\right\}$ and assume that the labeling is such that $s_{1} s_{2}$ and $s_{3} s_{4}$ have order 3 , and $s_{2} s_{3}$ has order 4. As in the preceding paragraph we will identify $s_{i}$ with $i(i=1,2,3,4)$. Then

$$
X \backslash X^{\prime}=\{2321,12321,232,323,4323,3234,43234\},
$$

and it follows from (4.7) that $n_{w}=1$ whenever $w \in \Delta_{s, t} \cap\left(X \backslash X^{\prime}\right)$.

For the rest of this section we will assume that $W$ is of type $\mathrm{B}_{l}$ and the labeling is as follows: $\left\{\alpha_{1}, \ldots, \alpha_{l}\right\}$ is a base of a root system of type $\mathbf{B}_{l}$; for $1 \leq i \leq l, s_{i}$ is the reflection corresponding to $\alpha_{i}$; and

$$
\left\langle\alpha_{i}, \alpha_{j}\right\rangle=\left\{\begin{array}{cl}
-2 & \text { if }(i, j)=(l-1, l), \\
-1 & \text { if }|i-j|=1 \text { and }(i, j) \neq(l-1, l), \\
0 & \text { otherwise. }
\end{array}\right.
$$

Notice that this notation is consistent with that of $\S 3$. 
Let $j$ and $k$ be such that $1 \leq j \leq k \leq l$. Define $x_{j, k}=s_{j} s_{j+1} \cdots s_{k}$ (here $\left.x_{j, j}=s_{j}\right)$, and define $x_{k, j}=x_{j, k}^{-1}$. Then

$$
X^{\prime}=\left\{x_{j, k} \mid 1 \leq j, k \leq l\right\} .
$$

Let $j$ and $k$ be such that $1 \leq j, k \leq l-1$. Define

$$
y_{j, k}=s_{j} s_{j+1} \cdots s_{l} \cdots s_{k},
$$

and define $z_{l}=s_{l} s_{l-1} s_{l}$. Notice that $y_{j, k}^{-1}=y_{k, j}$. We can now state

(4.4) Proposition. $X \backslash X^{\prime}=\left\{y_{j, k} \mid 1 \leq j, k \leq l-1\right\} \cup\left\{z_{l}\right\}$.

The proof of (4.4) depends on the following lemma.

(4.5) Lemma. Let $1 \leq j, k \leq l-1$. Then $\mathscr{R}\left(y_{j, k}\right)=\left\{s_{k}\right\}$, and $\ell\left(y_{j, k}\right)=$ $2 l-j-k+1$.

Proof. In the notation of $\S 3, y_{j, k}$ is the signed permutation

$$
(1, \ldots, j-1, j+1, \ldots, k,-j, k+1, \ldots, l)
$$

of $\{1, \ldots, l\}$. It follows easily from (3.1) that $\mathscr{R}\left(y_{j, k}\right)=\left\{s_{k}\right\}$. The fact that $\ell\left(y_{j, k}\right)=2 l-j-k+1$ now follows by induction on $l-k$. This proves (4.5).

We now prove the containment $\supseteq$ in (4.4). Clearly $z_{l}$ has a unique reduced expression and $z_{l} \notin X^{\prime}$, so by (2.5(4)), $z_{l} \in X-X^{\prime}$. Let $j$ and $k$ be such that $1 \leq j, k \leq l-1$. Then it follows from (4.5), and the definition of $\leq_{R}$ (see $[8])$, that

$$
s_{j} \cdots s_{l} \sim_{R} s_{j} \cdots s_{l} s_{l-1} \sim_{R} \cdots \sim_{R} s_{j} \cdots s_{l} \cdots s_{k} .
$$

Hence $y_{j, k} \in X \backslash X^{\prime}$.

To prove the opposite containment in (4.4), it clearly suffices to prove

$$
\begin{aligned}
& \text { If } Y=\left\{x_{j, k} \mid 1 \leq j, k \leq l\right\} \cup\left\{y_{j, k} \mid 1 \leq j, k \leq\right. \\
& l-1\} \cup\left\{z_{l}\right\}, \text { then } X \subseteq Y .
\end{aligned}
$$

Let $w \in X$; we will show by induction on $\ell(w)$ that $w \in Y$. If $\ell(w)=1$, then $w=s_{j}=x_{j, j}$ for some $j$, so $w \in Y$. Suppose $\ell(w)>1$ and $\mathscr{R}(w)=$ $\left\{s_{i}\right\}$ (recall that $w \in X$ ). By induction, $w s_{i} \in Y$ because $w s_{i} \in X$. There are several cases.

First, suppose that $w s_{i}=z_{l}$. Then $w=z_{l} s_{i}=s_{l} s_{l-1} s_{l} s_{i}$, and so no matter what $i$ is, $w$ does not have a unique reduced expression. Therefore by $(2.5(4))$, $w \notin X$, a contradiction. Hence $w s_{i} \neq z_{l}$.

Next, suppose that $w s_{i}=x_{j, k}$, where $j \leq k$. Then $w=x_{j, k} s_{i} \in X$. By (2.5(4)), $w$ has a unique reduced expression, so we may conclude that either $k<l$ and $i=k+1$, in which case $w=x_{j, k+1}$, or $k=l$ and $i=l-1$, in which case $w=y_{j, l-1}$. In either case, $w \in Y$.

If $w s_{i}=x_{j, k}$ with $k \leq j$, then an argument similar to the preceding shows that $w \in Y$. 
Finally, suppose that $w s_{i}=y_{j, k}$ for some $j, k$. Then $w=y_{j, k} s_{i} \in X$, and it follows from (2.5(4)) that $k>1$ and $i=k-1$, so $w=y_{j, k-1} \in Y$.

Since the preceding cases are exhaustive, it follows that (4.6) holds and hence that (4.4) is proved.

We conclude this section by showing that $n_{w}=1$ whenever $s$ and $t$ are in $S$ and $w$ is in $\Delta_{s, t} \cap\left(X \backslash X^{\prime}\right)$.

(4.7) Proposition. Let $s, t \in S$ and $w \in \Delta_{s, t} \cap\left(X-X^{\prime}\right)$. Then $n_{w}=1$.

To prove (4.7) we need to express the basis elements $C_{y}$, for $y \in\left\{y_{j, k} \mid 1 \leq\right.$ $j, k \leq l-1\}$, in terms of the generators $\left\{C_{s} \mid s \in S\right\}$ of $\mathscr{H}$.

(4.8) Lemma. Fix $j, k$ with $1 \leq j \leq l-1$ and $2 \leq k \leq l-1$, and put $y=y_{j, k}$. Let $z<y$. Then either $z s_{k}>z$ or $z s_{k-1}>z$.

Proof. If $j \geq k$ then $z s_{k-1}>z$, and the result holds. Hence we may assume that $j<k$. Suppose that $z s_{k-1}<z$, we will show that $z s_{k}>z$. By the Subword Property, $z$ is obtained by deletion from $y=s_{j} \ldots s_{l} \cdots s_{k}$. Thus $z s_{k-1}<z$ implies $s_{k-1}$ is not deleted. There are several cases.

First, suppose that both $s_{k}$ 's are deleted. Then clearly $z s_{k}>z$.

Next, suppose that the first $s_{k}$ is deleted. In this case, move $s_{k-1}$ as far to the right as possible in $z$. Then $z=w s_{k-1} s_{k}$, where $s_{k-1} \not z w, s_{k} \not w$, and $\ell(z)=\ell(w)+2$. Since $z s_{k-1}<z$, we have $w s_{k-1} s_{k} s_{k-1}<w s_{k-1} s_{k}$. By the Exchange Property, either

(1) $w s_{k-1} s_{k} s_{k-1}=\hat{w} s_{k-1} s_{k}$ (where $\hat{w}<w$ and $\left.\ell(\hat{w})=\ell(w)-1\right)$,

(2) $w s_{k-1} s_{k} s_{k-1}=w s_{k}$, or

(3) $w s_{k-1} s_{k} s_{k-1}=w s_{k-1}$.

The last two possibilities cannot occur because $\left|s_{k-1} s_{k}\right|>2$. Therefore

$$
w s_{k-1} s_{k} s_{k-1}=\hat{w} s_{k-1} s_{k},
$$

so $w s_{k}=\hat{w}<w$. But this contradicts our assumption that $s_{k} \not \leq w$, hence this case cannot occur.

Next, suppose that the second $s_{k}$ is deleted. Then

$$
z \leq s_{j} \cdots s_{k-1} s_{k} \cdots s_{l} \cdots s_{k+1} .
$$

In this case, considering $z$ as a permutation, we have $z(k-1)=k$ and $z(k)=$ $k+1$. Then by (3.1), $z s_{k-1}>z$, a contradiction. Hence this case cannot occur.

Finally, suppose that no $s_{k}$ 's are deleted. Then

$$
z \leq s_{j} \cdots s_{k-1} s_{k} \cdots s_{l} \cdots s_{k} \text {. }
$$

Again, considering $z$ as a permutation, we have $z(k-1)=k$. Since $z s_{k-1}<z$, it follows from (3.1) that $k \notin N(z)$. Hence $z(k)<z(k-1)$. It is easily checked that $z(k+1)=k+1$, so $z(k)<z(k+1)$. Using (3.1) one more time, we see that $z s_{k}>z$.

Because the previous cases are exhaustive, it follows that (4.8) is proved. 
In order to simplify the notation, we will write $C_{i}$ for $C_{s}$ when $s=s_{i}$. Also, given $j, k$ with $1 \leq j \leq k \leq l$, define $D_{j, k}$ to be $C_{j} C_{j+1} \cdots C_{k}$, and define $D_{k, j}$ to be $C_{k} C_{k-1} \cdots C_{j}$. Notice that $D_{j, j}=C_{j}$ for $1 \leq j \leq l$.

(4.9) Lemma.

(1) Let $j$ be such that $1 \leq j \leq l-1$ and let $y=y_{j, l-1}$. Then

$$
C_{y}=D_{j, l-1} C_{l} D_{l-1, l-1}-D_{j, l-1} \text {. }
$$

(2) Let $j$ and $k$ be such that $1 \leq j \leq l-1$ and $1 \leq k \leq l-2$, and let $y=y_{j, k}$. Then

$$
C_{y}=D_{j, l-1} C_{l} D_{l-1, k}-D_{j, l-1} D_{l-2, k}
$$

(here we have assumed that $l>2$ ).

Proof. (1) Let $y=y_{j, l-1}$, and put $x=x_{j, l}$. Then by (4.1), $C_{x}=D_{j, l}$, and by (2.1),

$$
C_{x} C_{l-1}=C_{y}+\sum_{\substack{z<x \\ z s<s}} \mu(z, x) C_{z},
$$

where we have written $s$ for $s_{l-1}$.

We claim that when $z<x, z s_{l-1}<z$, and $\mu(z, x) \neq 0$, we must have that $z=x s_{l}$. If so, then by (4.1), $C_{z}=D_{j, l-1}$, and (1) follows from (4.10). Just suppose that $z \neq x s_{l}$. Then by $(2.9), \mathscr{R}(x) \subseteq \mathscr{R}(z)$, so $z s_{l}<z$. Since $z s_{l-1}<z$ and $x=s_{j} \cdots s_{l}$, it follows from the Subword Property that $z=$ $w s_{l-1} s_{l}$, where $w \leq s_{j} \cdots s_{l-2}$. But then $z s_{l-1}>z$, a contradiction. Hence $z=x s_{l}$, and (a) is proved.

(2) We will prove (2) using induction on $l-k$. Suppose that $k=l-2$, and let $y=y_{j, l-1}$. By $(2.1)$, we have

$$
C_{y} C_{l-2}=C_{y s}+\sum_{\substack{z<y \\ z s<z}} \mu(z, y) C_{z},
$$

where we have written $s$ for $s_{l-2}$.

We claim that there are no $z \in W$ with $z<y, z s_{l-2}<z$, and $\mu(z, y) \neq 0$. Hence the result follows from (4.11) and (1).

Just suppose that $z \in W$ with $z<y, z s_{l-2}<z$, and $\mu(z, y) \neq 0$. By (4.8), $z s_{l-1}>z$. Since $z s_{l-2}<z$, it follows that $z \neq y s_{l-1}=s_{j} \cdots s_{l}$. Then by (2.9), $\mathscr{R}(y) \subseteq \mathscr{R}(z)$. Therefore $z s_{l-1}<z$, a contradiction, so no such $z$ can exist.

Next, suppose that $k<l-2$, and let $y=y_{j, k+1}$. By (2.1) we have

$$
C_{y} C_{k}=C_{y s}+\sum_{\substack{z<y \\ z s<z}} \mu(z, y) C_{z}
$$

where we have written $s$ for $s_{k}$. The argument used in the preceding paragraph shows that the sum in (4.12) is empty, so (2) follows easily by induction. This completes the proof of $(4.9)$. 
We can now prove (4.7). It follows from (4.9) and (2.6), that if $1 \leq j, k \leq$ $l-1, y=y_{j, k}, s=s_{k}$, and $t=s_{j}$, then $C_{y} d_{k}=-\left(u^{1 / 2}+u^{-1 / 2}\right) d_{j}$. Therefore $n_{y}=1$. Hence, to prove (4.7) it remains to show that if $z=z_{l}$ and $s=s_{l}$, then $n_{z}=1$. This last statement is an easy consequence of the fact that $C_{z}=$ $C_{l} C_{l-1} C_{l}-C_{l}$, which is proved using (2.1). This completes the proof of (4.7).

\section{Application to type $\mathrm{A}_{l-1}$}

In this section we apply the results of the previous sections to give explicit formulas for the $\pi(x, s, t)$ when $W$ is of type $\mathrm{A}_{l-1}$. In order to do this, we first compute the Kazhdan-Lusztig polynomials $Q_{x, w}$, for $x \in X$ and $w \in$ $W$. These polynomials have also been computed using a different method by Lascoux and Schutzenberger [7]. We conclude the section by deriving an elegant formula for the diagonal entries, $\pi(x, s, s)$, due to Tiwari [14]. In $\S 6$, we will consider the case in which $W$ is of type $\mathrm{B}_{l}$. This latter case is complicated by the fact that the Bruhat posets arising from the double coset spaces of pairs of maximal parabolic subgroups are not linearly ordered, as they are when $W$ is of type $A_{l-1}$.

Throughout this section we will assume $W$ is of type $\mathrm{A}_{l-1}$ and use the results and notation of $\S 3$ and $\S 4$. In particular, we will consider elements of $W$ as permutations of the set $\{1, \ldots, l\}$.

Recall that for $1 \leq j, k \leq l-1, A_{j}$ is the maximal parabolic subgroup generated by $\left\{s_{i} \mid 1 \leq i \leq l-1\right.$ and $\left.i \neq j\right\}$. Also, when $j \leq k$, the minimal $\left(A_{j}, A_{k}\right)$ double coset representatives are $\left\{x_{a, 0}^{j, k} \mid j+k-l \leq a \leq j\right\}$, where $x_{a, 0}^{j, k}$ is defined as in (3.6). When $j$ and $k$ are fixed, we will write $y_{a}$ for $x_{a, 0}^{j, k}$. Since $w \in W$ implies that $\nu_{j, k}(w)=(a, 0)$ for some $a$, we will write just $\nu_{j, k}(w)=a$ throughout this section. Notice that $y_{j}=1, y_{j-1}=s_{j} \cdots s_{k}$, and if $j=k$, then $y_{j-1}=s_{j}$.

By the results in $\S 4, X=\left\{x_{j, k} \mid 1 \leq j, k \leq l-1\right\}$, where in the notation of the preceding paragraph, $x_{j, k}=y_{j-1}=s_{j} \cdots s_{k}$.

(5.1) Proposition. Suppose that $1 \leq j \leq k \leq l-1, w \in W, \nu_{j, k}(w)=a$ with $a \leq j-1$ and $x=x_{j, k}$. Then $Q_{x, w}=1+u+\cdots+u^{j-a-1}$.

Proof. By [6, (2.3g)], the function $Q_{x,-}$ from $W$ to $\mathbb{Q}[u]$ is constant on $\left(A_{j}, A_{k}\right)$ double cosets, so we may assume that $w=y_{a}$ is a minimal $\left(A_{j}, A_{k}\right)$ double coset representative. We will prove the result using induction on $j$.

First, suppose that $j=1$; then $a=0$. Hence $x=w$, so $Q_{x, w}=1$ as desired.

Next, suppose that $j=2$, and write $s$ for $s_{1}$. If $a=1$, then $x=w$ and $Q_{x, w}=1$, so the result holds. If $a=0$, then from (2.10) we have

$$
Q_{x, w}=Q_{s x, s w}+u Q_{s x, w} .
$$


Notice that $s x=x_{1, k}$. It follows from the definitions (and our assumption that $\left.w=y_{0}\right)$ that $\nu_{1, k}(s w)=\nu_{1, k}(w)=0$. Thus, by the preceding paragraph, $Q_{s x, s w}=Q_{s x, w}=1$. Therefore $Q_{x, w}=1+u$, and so (5.1) holds.

Finally, suppose that $j>2$. If $a=j-1$, then $x=w$, so $Q_{x, w}=1$. Assume that $a<j-1$, and put $s=s_{j-1}$ and $t=s_{j-2}$. Then by (2.10),

$$
Q_{x, w}=Q_{s x, s w}+u Q_{s x, w}-u Q_{t s x, w} .
$$

Notice that $s x=x_{j-1, k}$ and $t s x=x_{j-2, k}$. Thus, we can compute $Q_{s x, s w}$, $Q_{s x, w}$, and $Q_{t s x, w}$ once we determine $\nu_{j-1, k}(s w), \nu_{j-1, k}(w)$, and $\nu_{j-2, k}(w)$. It follows from the definitions that

$$
\nu_{j-1, k}(s w)=\nu_{j-1, k}(w)=\nu_{j-2, k}(w)=a .
$$

If $a=j-2$, then $t s x \notin z$; so by (5.3) and induction, $Q_{x, w}=1+u$. If $a<j-2$, then by (5.3) and induction,

$$
\begin{aligned}
Q_{x, w} & =\left(1+\cdots+u^{j-a-2}\right)+u\left(1+\cdots+u^{j-a-2}\right)-u\left(1+\cdots+u^{j-a-3}\right) \\
& =1+\cdots+u^{j-a-1} .
\end{aligned}
$$

This completes the proof of (5.1).

We can now compute the matrix entries $\pi(y, s, t)$ for $y \in W$. Let $y$ be in $W$ and $s$ be in $S$. It follows from (1.6) that $\pi(y, s, t)=-\pi(y s, s, t)$, so we may assume that $y s>y$. In order to simplify the notation somewhat, we will write $\pi(y, k, j)$ for $\pi(y, s, t)$ when $s=s_{k}$ and $t=s_{j}$.

(5.4) Theorem. Suppose $1 \leq j, k \leq l-1$ and $y \in W$ with $y s_{k}>y$ and $\nu_{j, k}(y)=a$. Then

$$
\pi(y, k, j)= \begin{cases}u^{l(y)+a-(1 / 2)(j+k)} & \text { if } \nu_{j, k}\left(y s_{k}\right)=a-1, \\ 0 & \text { otherwise. }\end{cases}
$$

Proof. We first remark that $y s_{k}>y$ implies that either $\nu_{j, k}\left(y s_{k}\right)=a$, or $\nu_{j, k}\left(y s_{k}\right)=a-1$.

Let $s=s_{k}, t=s_{j}$, and $x=x_{j, k}$. It follows from the results in $\S 4$ that $\Delta_{s, t}=\{x\}$ and that $n_{x}=1$. Hence we may rewrite (2.8) as

$$
\pi(y, k, j)=u_{y}\left[\overline{u^{|j-k| / 2}\left(Q_{x, y s}-Q_{x, y}\right)}\right] .
$$

If $\nu_{j, k}(y s)=\nu_{j, k}(y)$, then $Q_{x, y s}=Q_{x, y}$, so $\pi(y, k, j)=0$.

Let $\nu_{j, k}(y s)=a-1$. To complete the proof it suffices to prove

$$
Q_{x, y s}-Q_{x, y}=u^{m-a}
$$

where $m=\min \{j, k\}$.

Suppose first that $m=j \leq k$ and $a=j$. Then $Q_{x, y}=Q_{x, 1}=0$ and $Q_{x, y s}=Q_{x, x}=1$, so (5.5) holds. If $m=j$ and $a<j$, then (5.5) follows from (5.1). 
Next suppose that $m=k<j$, then

$$
Q_{x, y s}-Q_{x, y}=Q_{x^{-1}, s y^{-1}}-Q_{x^{-1}, y^{-1}} .
$$

Since $\nu_{k, j}\left(w^{-1}\right)=\nu_{j, k}(w)$ for all $w \in W$, it follows from the preceding paragraph that the right-hand side of (5.6) is $u^{k-a}=u^{m-a}$. Hence (5.5) is proved.

We conclude this section, as mentioned above, by deriving from the preceding discussion a formula due to Tiwari for the diagonal entries, $\pi(y, i, i)$. Unfortunately (?) this formula does not seem to generalize to other types of Weyl groups.

Let $j$ and $k$ be such that $1 \leq i \leq l-1$ and let $x \in W$. Define $\ell_{i}(x)$ to be the minimal number of $s_{i}$ 's appearing in a reduced expression for $x$. For example, $\ell_{i}\left(s_{1} s_{2} s_{1}\right)=1$, since $s_{1} s_{2} s_{1}=s_{2} s_{1} s_{2}$. We will say that a reduced expression for $x$ is $i$-reduced if the number of $s_{i}$ 's it contains is exactly $\ell_{i}(x)$.

For the rest of this section, fix $i$ with $1 \leq i \leq l-1$, and write $\nu$ for $\nu_{i, i}$. Thus

$$
\nu(x)=|\{1, \ldots, i\} \cap x(\{1, \ldots, i\})| .
$$

(5.7) Proposition. Let $1 \leq i \leq l-1$, and let $x \in W$. Then $\ell_{i}(x)=i-\nu(x)$.

Proof. We first show that $\ell_{i}(x)$ is constant on $\left(A_{i}, A_{i}\right)$ double cosets. Suppose that there is a $k$ with $k \neq i$ and $s_{k} x<x$. Let $x=s_{p_{1}} \cdots s_{p_{r}}$ be an $i$-reduced expression for $x$. Then by the Exchange Property, $s_{k} x=s_{p_{1}} \cdots \widehat{p_{j}} \cdots s_{p_{r}}$, for some $j$, where - means delete. Because $k \neq i$, it follows that $p_{j} \neq i$, so $s_{k} s_{p_{1}} \cdots \widehat{s_{p_{j}}} \cdots s_{p_{r}}$ is $i$-reduced. Hence $\ell_{i}(x) \leq \ell_{i}\left(s_{k} x\right)$. Also, $\ell_{i}\left(s_{k} x\right) \leq \ell_{i}(x)$ because $k \neq i$, so $\ell_{i}(x)=\ell_{i}\left(s_{k} x\right)$. It follows that $\ell_{i}(x)$ depends only on the $\left(A_{i}, A_{i}\right)$ double coset containing $x$.

Recall that the minimal $\left(A_{i}, A_{i}\right)$ double coset representatives are $\left\{y_{a} \mid 2 i-\right.$ $l \leq a \leq i\}$, and that $\nu\left(y_{a}\right)=a$. We will show, using induction on $i-a$, that $\ell_{i}\left(y_{a}\right)=i-a$.

If $a=i$, then $y_{i}=1$, and there is nothing to prove. Assume that $a<i$ and $\ell_{i}\left(y_{a+1}\right)=i-a-1$. It follows from the definitions that $\nu\left(s_{i} y_{a+1}\right)=a$, so it suffices to show that $\ell_{i}\left(s_{i} y_{a+1}\right)=\ell_{i}\left(y_{a+1}\right)-1$. This follows from

(1) $\mathscr{L}\left(y_{a+1}\right)=\left\{s_{i}\right\}$, and

(2) if $w \in W$ with $s_{i} w<w$, then $\ell_{i}(w)-1 \leq \ell_{i}\left(s_{i} w\right) \leq \ell_{i}(w)$.

(1) follows from the fact that $y_{a+1} \in X_{i, i}$, and (2) is proved using the Exchange Property, as in the preceding paragraph. This completes the proof of (5.7).

Let $y \in W$. Define $r_{y}$ to be the coefficient of the simple root $\alpha_{i}$ in $y\left(\alpha_{i}\right)$, Then $r_{y} \in\{-1,0,1\}$. Also, define $m(y)$ to be $\ell(y)-\ell_{i}(y)$.

(5.8) Proposition. Let $y \in W$. Then $\pi(y, i, i)=r_{y} u^{m(y)}$. 
Proof. Write $s$ for $s_{i}$ and suppose that $y(i)=j_{1}$ and $y(i+1)=j_{2}$ (here we consider $y$ as a permutation). Then

$$
r_{y}=\left\{\begin{array}{cl}
1 & \text { if } j_{1} \leq i \leq j_{2}-1 \\
-1 & \text { if } j_{2} \leq i \leq j_{1}-1 \\
0 & \text { otherwise }
\end{array}\right.
$$

and $y s>y$ if and only if $j_{1}<j_{2}$.

First, suppose that $y s>y$. Then $r_{y} \in\{0,1\}$. If $r_{y}=0$ then either $i<j_{1}$, or $j_{2} \leq i$. Hence

$$
\left\{j_{1}, j_{2}\right\} \cap\{1, \ldots, i\}= \begin{cases}\left\{j_{1}, j_{2}\right\} & \text { if } j_{2} \leq i \\ \varnothing & \text { otherwise }\end{cases}
$$

In either case, $\nu(y s)=\nu(y)$, so by $(5.4), \pi(y, i, i)=0$. Therefore, (5.8) is proved in this case. If $r_{y}=1$, then $\nu(y s)=\nu(y)-1$; so by $(5.4), \pi(y, i, i)=$ $u^{\ell(y)+a-i}$ where $a=\nu(y)$. By $(5.7), \nu(y)=i-\ell_{i}(y)$, so (5.8) holds in this case also.

Next, suppose that $y s<y$. Then $r_{y} \in\{-1,0\}$ and $j_{2}<j_{1}$. If $r_{y}=0$, then either $i<j_{2}$ or $j_{1} \leq i$. In either case, $\nu(y s)=\nu(y)$; so by (5.4), $\pi(y, i, i)=-\pi(y s, i, i)=0$. Finally, if $r_{y}=-1$, then $\nu(y s)=\nu(y)+1$, so $m(y)=m(y s)$. Because $r_{y}=-r_{y s}$, it follows from the preceding paragraph that $\pi(y, i, i)=-\pi(y s, i, i)=r_{y} u^{m(y)}$. This completes the proof of (5.8).

\section{Application to Type B,}

Throughout this section, $W$ will be a Weyl group of type $\mathrm{B}_{l}$, and we will use the notation of $\S 3$ and $\S 4$. In particular,

$$
X=\left\{x_{j, k} \mid 1 \leq j, k \leq l\right\} \cup\left\{y_{j, k} \mid 1 \leq j, k \leq l-1\right\} \cup\left\{z_{l}\right\} .
$$

We will also write $\pi(y, k, j)$ for $\pi(y, s, t)$, and $\Delta_{k, j}$ for $\Delta_{s, t}$, when $s=s_{k}$ and $t=s_{j}$. In this section we compute the Kazhdan-Lusztig polynomials, $Q_{x, w}$, for $x \in X$ and $w \in W$, and the matrix entries, $\pi(y, k, j)$, for $y \in w$ and $1 \leq j, k \leq l$. As in $\S 5, \pi(y, k, j)$ depends only on $\nu_{j, k}(y)$ and $\nu_{j, k}\left(y s_{k}\right)$; but if $\nu_{j, k}(y)=(a, b)$, and $y s_{k}>y$, there are four possibilities for $\nu_{j, k}\left(y s_{k}\right)$. In each of these cases we obtain a different formula for $\pi(y, k, j)$.

(6.1) Proposition. Let $j$ and $k$ be such that $1 \leq j, k \leq l$. Let $x \in \Delta_{j, k}$, and let $w \in W$ with $\nu_{j, k}(w)=(a, b)$.

(1) Suppose $1 \leq j<l, x=x_{j, l}$ and $a \leq j-1$. Then

$$
Q_{x, w}=1+u+\cdots+u^{j-a-1} \text {. }
$$

(2) Suppose $1 \leq j \leq k \leq l-1, x=x_{j, k}$ and $a \leq j-1$. Then

$$
Q_{x, w}=1+u+\cdots+u^{j-a-1}
$$


(3) Suppose $x=z_{l}$ (so $j=k=l$ ) and $a \leq l-2$. Then

$$
Q_{x, w}=1+u^{2}+u^{4}+\cdots+u^{2[(l-a-2) / 2]}
$$

where $[\cdot]$ is the greatest integer function.

(4) Suppose $x=s_{l}($ so $j=k=l)$ and $a \leq l-1$. Then

$$
Q_{x, w}=1+u^{2}+\cdots+u^{2[(l-a-1) / 2]} .
$$

(5) Suppose $1 \leq j \leq k \leq l-1, x=y_{j, k}$ and $b \geq 1$. Then

$$
Q_{x, w}=1+u+\cdots+u^{b-1} .
$$

Proof. The proof of (6.1) is long, but straightforward, and the arguments are the same as those used in the proof of (5.1). For example, (1) is proved using induction on $j$. If $j=1$ then there is nothing to prove. Let $j \geq 2, y \in W$, and suppose $I=S-\mathscr{L}(y)$ and $J=S-\mathscr{R}(y)$. Then $Q_{y,-}$ is constant on $\left(W_{I}, W_{J}\right)$ double cosets. This fact, and the recursion formula in (2.10) are used to express $Q_{x, w}$ in terms of Kazhdan-Lusztig polynomials, $Q_{x^{\prime}, w^{\prime}}$, where the " $j$ " for $x^{\prime}$ is " $j-1$." We omit further details of the proof.

Suppose for a moment that $1 \leq j \leq k \leq l-1, y \in W$ with $y s_{k}>y$, and $\nu_{j, k}(y)=(a, b)$. Considering $y$ as a signed permutation, and using (3.1), there are three possibilities for $y(k)$ and $y(k+1)$ :

(1) $k, k+1 \notin N(y)$ and $y(k)<y(k+1)$,

(2) $k, k+1 \in N(y)$ and $y(k+1)<y(k)$, or

(3) $k \notin N(y)$ and $k+1 \in N(y)$.

Suppose (1) holds. Then either $y(k) \leq j \leq y(k+1)$ or not. If so, then $\nu_{j, k}\left(y s_{k}\right)=(a-1, b)$, and if not, then $\nu_{j, k}\left(y s_{k}\right)=(a, b)$.

Suppose (2) holds. Then either $y(k+1) \leq j \leq y(k)$ or not. If so, then $\nu_{j, k}\left(y s_{k}\right)=(a, b+1)$, and if not, then $\nu_{j, k}\left(y s_{k}\right)=(a, b)$.

Suppose (3) holds. Then there are four cases:

(i) If $y(k)>j$ and $y(k+1)>j$, then $\nu_{j, k}\left(y s_{k}\right)=(a, b)$.

(ii) If $y(k) \leq j$ and $y(k+1)>j$, then $\nu_{j, k}\left(y s_{k}\right)=(a-1, b)$.

(iii) If $y(k)>j$ and $y(k+1) \leq j$, then $\nu_{j, k}\left(y s_{k}\right)=(a, b+1)$.

(iv) If $y(k) \leq j$ and $y(k+1) \leq j$, then $\nu_{j, k}\left(y s_{k}\right)=(a-1, b+1)$.

We may conclude that, in any case,

$$
\nu_{j, k}\left(y s_{k}\right) \in\{(a, b),(a-1, b),(a, b+1),(a-1, b+1)\},
$$

and all four possibilities can occur.

Let $j$ be such that $1 \leq j \leq l$, and let $y \in W$ with $y s_{l}>y$. Suppose $\nu_{j, l}\left(y s_{l}\right)=(a, b)$. Then a similar argument shows

$$
\nu_{j, l}\left(y s_{l}\right) \in\{(a, b),(a-1, b+1)\} .
$$


(6.2) Proposition. Suppose $1 \leq j, k \leq l, y \in W$ with $y s_{k}>y$, and $\nu_{j, k}(y)=$ $(a, b)$.

(1) Let $j$ and $k$ be such that $1 \leq j, k \leq l-1$. Then

$$
\pi(y, k, j)= \begin{cases}0 & \text { if } \nu_{j, k}\left(y s_{k}\right)=(a, b), \\ u^{\ell(y)+a-(1 / 2)(j+k)} & \text { if } \nu_{j, k}\left(y s_{k}\right)=(a-1, b), \\ u^{\ell(y)+j+k-2 l-b} & \text { if } \nu_{j, k}\left(y s_{k}\right)=(a, b+1), \\ u^{\ell(y)}\left(u^{a-(1 / 2)(j+k)}+u^{j+k-2 l-b}\right) & \text { if } \nu_{j, k}\left(y s_{k}\right)=(a-1, b+1) .\end{cases}
$$

(2) Let $j$ be such that $1 \leq j \leq l$. Then

$$
\pi(y, l, j)= \begin{cases}0 & \text { if } \nu_{j, l}\left(y s_{l}\right)=(a, b), \\ u^{\ell(y)+a-(1 / 2)(j+l)} & \text { if } \nu_{j, l}\left(y s_{l}\right)=(a-1, b+1),\end{cases}
$$

(3) Let $k$ be such that $1 \leq k \leq l$. Then

$$
\pi(y, k, l)= \begin{cases}0 & \text { if } \nu_{l, k}\left(y s_{k}\right)=(a, b), \\ 2 u^{\ell(y)+a-(1 / 2)(k+l)} & \text { if } \nu_{l, k}\left(y s_{k}\right)=(a-1, b+1) .\end{cases}
$$

Proof. Let $j$ and $k$ be such that $j \leq k$. Then (6.2) is a straightforward application of (2.8), the computation of the $n_{w}(w \in X)$ in $\S 4,(6.1)$, and the preceding remarks concerning $\nu_{j, k}\left(y s_{k}\right)$.

Let $j$ and $k$ be such that $j>k$. Then (6.2) is proved as in the previous case, using the additional results: $Q_{x, w}=Q_{x^{-1}, w^{-1}}$, for $x, w \in W$; and $\nu_{j, k}(y)=$ $\nu_{k, j}\left(y^{-1}\right)$, for $y \in W$.

As an example, we will prove (2) when $j=k=l$ (this is the most complicated case). Let $s=s_{l}$ and $z=z_{l}$. Then, by the results in $\S 4, \Delta_{l, l}=\{s, z\}$ and $n_{s}=n_{z}=1$. Hence, by (2.8), it suffices to prove

$$
\text { If } \nu_{l, l}\left(y s_{l}\right)=(a-1, b+1) \text {, then } \pi(y, l, l)=u^{\ell(y)+a-l} .
$$

Using (2.8) again, we have

$$
\pi(y, l, l)=u_{y}\left[\overline{\left(Q_{s, y s}-Q_{s, y}\right)+u\left(Q_{z, y s}-Q_{z, y}\right)}\right] .
$$

If $a=l$, then $Q_{s, y s}=1$ and $Q_{s, y}=Q_{z, y s}=Q_{z, y}=0$; so (6.4) implies (6.3). If $a=l-1$, then $Q_{s, y s}=Q_{s, y}=Q_{z, y s}=1$ and $Q_{z, y}=0$; so (6.4) implies (6.3) in this case also. If $a \leq l-2$ and $l-a$ is even, then by (6.1) and (6.4),

$$
\pi(y, l, l)=u_{y}\left[\overline{\left.u^{l-a}+0\right]}=u^{\ell(y)+a-l} .\right.
$$

If $a \leq l-2$ and $l-a$ is odd, then by (6.1) and (6.4),

$$
\pi(y, l, l)=u_{y}\left[\overline{0+u\left(u^{l-a-1}\right)}\right]=u^{\ell(y)+a-l} .
$$

Hence (6.3) holds, and so $(6.2(2))$ is proved. 


\section{REFERENCES}

1. A. Björner, Orderings of Coxeter groups, Combinatorics and Algebra (Boulder, Colo., 1983), Contemp. Math., vol. 34, Amer. Math. Soc., Providence, R.I., 1984.

2. B. Boe, Kazhdan-Lusztig polynomials for Hermitian symmetric spaces, Trans. Amer. Math. Soc. 309 (1988), 279-294.

3. C. Curtis, N. Iwahori, and R. Kilmoyer, Hecke algebras and characters of parabolic type of finite groups with $(B, N)$-pairs, Publ. Math. Inst. Haute Etudes Sci. 40 (1971), 81-116.

4. V. Deodhar, Some characterizations of Bruhat ordering on a Coxeter group and determination of the relative Mobius function, Invent. Math. 39 (1977), 187-198.

5. J. M. Douglass and B. Shelton, On the matrix coefficients of the reflection representation, Proc. Amer. Math. Soc. 105 (1989), 62-65.

6. D. Kazhdan and G. Lusztig, Representations of Coxeter groups and Hecke algebras, Invent. Math. 53 (1979), 165-184.

7. A. Lascoux and M. Schutzenberger, Polynômes de Kazhdan et Lusztig pour les grassmanniennes, Young tableaux and Schur functions in algebra and geometry (Torun, 1980), Asterisque 87 (1981), 249-266.

8. G. Lusztig, On a theorem of Benson and Curtis, J. Algebra 71 (1981), 490-498.

9. _ Unipotent characters of the symplectic and odd orthogonal groups over a finite field, Invent. Math. 64 (1981), 263-296.

10. Characters of reductive groups over a finite field, Ann. of Math. Studies, no. 107, Princeton Univ. Press, Princeton, N.J., 1984.

11. Cells in Affine Weyl groups I, Algebraic Groups and Related Topics, Advanced Studies in Pure Math., 6, Kinokuniya, Tokyo, and North-Holland, Amsterdam, 1985.

12. __ Cells in Affine Weyl groups II, J. Algebra 109 (1987), 536-548.

13. L_ Leading coefficients of character values of Hecke algebras, The Arcata Conference on Representations of Finite Groups (Arcata, Calif., 1986), Proc. Sympos. Pure Math., vol. 47, Amer. Math. Soc., Providence, R. I., 1987, pp. 235-262.

14. H. Tiwari, Reflection representations of Hecke algebras of certain Weyl groups, J. Algebra 120 (1989), 224-245.

Department of Mathematics, University of North Texas, Denton, Texas 76203-5116 